\title{
Characterization of Odor-Active Compounds of Ichang Lemon \\ (Citrus wilsonii Tan.) and Identification of its Genetic Interspecific Origin by DNA Genotyping.
}

Benoît Demarcq ${ }^{1}$, Margaux Cavailles ${ }^{1}$, Laetitia Lambert ${ }^{1}$, Christine Schippa ${ }^{1}$, Patrick Ollitrault ${ }^{2,3}$ and François Luro ${ }^{3}$

${ }^{1}$ V Mane Fils SA, 620 Route de Grasse, 06620 LE BAR SUR LOUP, France

${ }^{2}$ CIRAD, UMR AGAP, F-20230 San Giuliano, France

${ }^{3}$ UMR AGAP Institut, Université Montpellier, CIRAD, INRAE, Institut Agro -, 20230, San Giuliano, France

*Corresponding author

Phone: +33493097000

E-mail address: benoit.demarcq@mane.com

E-mail addresses of co-authors:

margaux.cavailles@mane.com

laetitia.lambert@mane.com

christine.schippa@mane.com

patrick.ollitrault@cirad.fr

francois.luro@inrae.fr 


\section{SUPPORTING INFORMATION}

Table S1. Description of the 27 SSRs and InDels markers.

\begin{tabular}{|c|c|c|c|c|c|c|c|c|}
\hline Locus Id & SSR type & Scaffold & $\begin{array}{l}\text { Position } \\
\text { (NT) }\end{array}$ & Forward primer sequence & Reverse primer sequence & $\mathbf{A T}^{\mathrm{a}}$ & Size range $^{b}$ & Ref. $^{c}$ \\
\hline IDEMA & Indel & 1 & 7406793 & СTCTTTCTGCTTCCTGACATC & GCCGGTGAATAAAACACAAC & 55 & 283-293 & 1 \\
\hline Ci01B10 & $(\mathrm{CT}) \mathrm{N}_{8}(\mathrm{~A})$ & 1 & 7328790 & AAAAATTGCCCTCTTCTCCT & TGGTGGTTTTGTTGGTTCTAT & 55 & $140-175$ & 2 \\
\hline Ci02D09 & (TC) & 2 & 5821030 & AATGATGAGGGTAAAGATG & ACCCATCACAAAACAGA & 55 & $241-265$ & 3 \\
\hline Ci04H06 & $(\mathrm{AG})$ & 2 & 8097528 & CAAAGTGGTGAAACCTG & GGACATAGTGAGAAGTTGG & 55 & $206-217$ & 4 \\
\hline MEST520 & $(\mathrm{AG}) \mathrm{N}_{4}(\mathrm{GGC})$ & 2 & 8450195 & CTCTGATATTCCCAAAGCCG & AAGCACGTTATCGGGATCTG & 55 & $204-289$ & 5 \\
\hline MEST375 & $($ TTTA $) /(\text { TTA })_{x}$ & 2 & 9066584 & CCCCCTTTTGTGATTGTTATG & GAAGGAAGAAAAAGAGACCAAAA & 55 & $140-193$ & 5 \\
\hline $\mathrm{Ci03C08}$ & (TC) & 2 & 27340105 & GCTTCTTACATTCCTCAAA & CAGAGACAGCCAAGAGA & 55 & $223-285$ & 4 \\
\hline Mest046 & (CAA) & 2 & 33532441 & GGTGAGCATCTGGACGACTT & GAACCAGAATCAGAACCCGA & 55 & $230-256$ & 6 \\
\hline $\mathrm{CAC} 23$ & $(\mathrm{CAC})$ & 3 & 209215 & TTGCATTGTAGCATGTTGG & ATCACAATTACTAGCAGCGCC & 55 & $265-281$ & 7 \\
\hline Mest131 & (GCCCCA) & 3 & 50550703 & GCTGTCACGTTGGGTGTATG & TACCTCCACGTGTCAAACCA & 55 & $147-177$ & 6 \\
\hline Ci01D06a & (CA) & 4 & 3481519 & GATCAAAACATTATTCCAA & TTTTTCATCAACAAGACTG & 50 & $225-257$ & 2 \\
\hline Ci07D06 & $(\mathrm{TC}) /(\mathrm{AC})$ & 4 & 7293957 & TCAATTCCTCTAGTGTGTGT & CCTTTTCACAGTTTGCTAT & 55 & $182-214$ & 3 \\
\hline IDPSY & Indel & 6 & 21393227 & CCTGTCGACATTCAGGTTAG & CTCATCACATCTTCGGTCTC & 55 & $263-266$ & 1 \\
\hline Mest192 & (AT) & 6 & 17474923 & CTTGGCACCATCAACACATC & CGCGGATCATCTAGCATACA & 55 & $217-259$ & 8 \\
\hline Mest488 & $(\mathrm{CT})$ & 6 & 21253842 & CTTTGCGTGTTTGTGCTGTT & CACGCTCTTGACTTTCTCCC & 55 & $135-165$ & 6 \\
\hline TAA1 & (TAA) & 6 & 24896288 & AAGAAGAAGAGCCCCCATTAGC & GACAACATCAACAACAGCAAGAGC & 55 & $161-180$ & 7 \\
\hline Mest107 & $(\mathrm{TCT}) \mathrm{N}_{6}(\mathrm{TC})$ & 7 & 211095 & CCCCATCCTTTCAACTTGTG & GCTGAGATGGGGATGAAAGA & 55 & $192-200$ & 6 \\
\hline Ci01C09 & (GA) & 7 & 3090384 & GACAGAATGGGAGAGGAGA & TTGTCCCTTCCCTTTGTA & 50 & $265-285$ & 2 \\
\hline MEST458 & (TC) & 7 & 6772689 & СССССТСТTТТТСТСТТССА & TTCTGGGCTGGTAGGTTCAG & 55 & $195-236$ & 3 \\
\hline Ci03B07 & (AC) & 7 & 11545537 & TGAGGGACTAAACAGCA & САССТTТСССТTССА & 55 & $269-289$ & 6 \\
\hline Ci07B05 & (GA) & 8 & 3217587 & TTTGTTCTTTTTGGTCTTTT & СTTTTCTTTCCTAGTTTCCC & 50 & $196-248$ & 2 \\
\hline $\mathrm{Ci02F07}$ & (AC) & 8 & 15057658 & TGCTGGTTTTCAGATACTT & GCAGCGTTTGTTTTCT & 55 & $187-229$ & 2 \\
\hline Mest015 & $(\mathrm{GAG})$ & 8 & 24850644 & GCCTCGCATTCTCTTGACTC & TTATTACGAAGCGGAGGTGG & 55 & $198-215$ & 6 \\
\hline IDLCY2 & Indel & 8 & 19261216 & CGCAAATAATTGATTCAACA & GATGATCACGTCATATCGAA & 50 & $237-246$ & 1 \\
\hline IDHYB1 & Indel & 9 & 29490771 & AAAAACAAAGCACCCAGAT & GCCACCAGAACCTGTAATAA & 53 & $216-231$ & 1 \\
\hline Ci07F11 & $(\mathrm{GA}) \mathrm{G}(\mathrm{GA})$ & 9 & 4918161 & ACTATGATTACTTTGCTTTGAG & GAAGAAACAAGAAAAAAAAAT & 50 & $140-180$ & 3 \\
\hline $\mathrm{Ci} 08 \mathrm{C} 05$ & (GA) & 9 & 25537858 & TCCACAGATTGCCCATTA & CCCTAAAAACCAAGTGACA & 55 & $126-180$ & 2 \\
\hline
\end{tabular}

a annealing temperature for $\mathrm{PCR} ;{ }^{\mathrm{b}}$ Range of amplified fragment sizes

c 1: Garcia-Lor et al., 2013; 2: Froelicher et al., 2008; 3: New; 4: Cuenca et al., 2011; 5: Ollitrault et al. 2012; 6: Garcia-Lor et al., 2012; 7: Kijas et al., 1997; 8: Aleza et al., 2011 
Table S2. Information on the DSNPs markers.

\begin{tabular}{|c|c|c|c|c|c|c|c|}
\hline Marker ID & Chrom. & Position & REF & ALT & Ancestor & Specific allele & Gene \\
\hline 1_112523 & 1 & 112523 & $T$ & $A$ & C. medica & A & Ciclev10007230m.g \\
\hline 1_429415 & 1 & 429415 & G & $A$ & C. ichangensis & $A$ & Ciclev10007367m.g \\
\hline 1_1202115 & 1 & 1202115 & G & A & C.maxima & A & Ciclev10010432m.g \\
\hline 1_1445049 & 1 & 1445049 & G & $T$ & C. reticulata & G & Ciclev10007276m.g \\
\hline 1_4381520 & 1 & 4381520 & $\mathrm{C}$ & G & C. micrantha & G & Ciclev10008549m.g \\
\hline 1_10558128 & 1 & 10558128 & C & $T$ & C.maxima & $T$ & Ciclev10010400m.g \\
\hline 1_11872921 & 1 & 11872921 & T & C & C. reticulata & $\mathrm{T}$ & Ciclev10007498m.g \\
\hline 1_12808366 & 1 & 12808366 & A & $\mathrm{T}$ & C. micrantha & $\mathrm{T}$ & Ciclev10010796m.g \\
\hline 1_12909134 & 1 & 12909134 & G & C & C. ichangensis & C & Ciclev10008033m.g \\
\hline 1_14599773 & 1 & 14599773 & $\mathrm{~T}$ & $\mathrm{C}$ & C. medica & $\mathrm{C}$ & Ciclev10007863m.g \\
\hline 1_23662492 & 1 & 23662492 & $\mathrm{C}$ & $\mathrm{T}$ & C. ichangensis & $T$ & Ciclev10007792m.g \\
\hline 1_24357413 & 1 & 24357413 & C & A & C.maxima & C & Ciclev10008979m.g \\
\hline 1_25122651 & 1 & 25122651 & A & G & C. reticulata & G & ET083949 \\
\hline 1_25274313 & 1 & 25274313 & T & A & C. micrantha & A & Ciclev10010780m.g \\
\hline 1_27714027 & 1 & 27714027 & $\mathrm{C}$ & A & C. medica & A & Ciclev10007428m.g \\
\hline 2_148798 & 2 & 148798 & $\mathrm{~A}$ & G & C. ichangensis & G & Ciclev10014977m.g \\
\hline 2_411531 & 2 & 411531 & $\mathrm{C}$ & $\mathrm{T}$ & C. reticulata & $\mathrm{C}$ & Ciclev10014253m.g \\
\hline 2_7448065 & 2 & 7448065 & G & C & C. medica & C & Ciclev10014020m.g \\
\hline 2_7659486 & 2 & 7659486 & C & G & C. micrantha & G & Ciclev10014019m.g \\
\hline 2_8056523 & 2 & 8056523 & A & $\mathrm{G}$ & C.maxima & $\mathrm{G}$ & Ciclev10018289m.g \\
\hline 2_11091624 & 2 & 11091624 & A & G & C. reticulata & $\mathrm{A}$ & Ciclev10014164m.g \\
\hline 2_11492295 & 2 & 11492295 & $\mathrm{~T}$ & C & C. ichangensis & C & Ciclev10014674m.g \\
\hline 2_15483255 & 2 & 15483255 & $\mathrm{C}$ & G & C.maxima & G & Ciclev10015371m.g \\
\hline 2_21022776 & 2 & 21022776 & A & G & C. micrantha & G & Ciclev10018135m.g \\
\hline 2_25198777 & 2 & 25198777 & G & A & C. medica & $\mathrm{A}$ & Ciclev10015267m.g \\
\hline 2_26306845 & 2 & 26306845 & $\mathrm{C}$ & $\mathrm{T}$ & C. reticulata & $\mathrm{C}$ & Ciclev10015265m.g \\
\hline 2_28936300 & 2 & 28936300 & A & C & C. ichangensis & C & Ciclev10014065m.g \\
\hline 2_31567713 & 2 & 31567713 & $\mathrm{C}$ & $\mathrm{T}$ & C. micrantha & $\mathrm{T}$ & Ciclev10018362m.g \\
\hline 2_31581849 & 2 & 31581849 & G & A & C.maxima & A & Ciclev10014154m.g \\
\hline 2_35686712 & 2 & 35686712 & G & $\mathrm{T}$ & C. medica & $\mathrm{T}$ & Ciclev10014760m.g \\
\hline 3_105647 & 3 & 105647 & A & G & C. ichangensis & $\mathrm{G}$ & Ciclev10018581m.g \\
\hline 3_165889 & 3 & 165889 & A & G & C.maxima & G & Ciclev10023360m.g \\
\hline 3_2073865 & 3 & 2073865 & $\mathrm{C}$ & $\mathrm{T}$ & C. micrantha & $\mathrm{T}$ & Ciclev10020017m.g \\
\hline 3_5866407 & 3 & 5866407 & C & G & C. reticulata & C & Ciclev10021633m.g \\
\hline 3_11355960 & 3 & 11355960 & A & G & C. medica & G & Ciclev10023509m.g \\
\hline
\end{tabular}




\begin{tabular}{|c|c|c|c|c|c|c|c|}
\hline 3_14861567 & 3 & 14861567 & $\mathrm{C}$ & $\mathrm{A}$ & C. micrantha & $\mathrm{A}$ & Ciclev10021253m.g \\
\hline 3_24894898 & 3 & 24894898 & $\mathrm{~T}$ & $\mathrm{C}$ & C. medica & $\mathrm{C}$ & Ciclev10019906m.g \\
\hline 3_27184394 & 3 & 27184394 & A & G & C.maxima & G & Ciclev10018865m.g \\
\hline 3_29352884 & 3 & 29352884 & $\mathrm{C}$ & $\mathrm{A}$ & C. reticulata & $\mathrm{C}$ & Ciclev10019364m.g \\
\hline 3_29381066 & 3 & 29381066 & $\mathrm{C}$ & $\mathrm{T}$ & C. ichangensis & $\mathrm{T}$ & Ciclev10019003m.g \\
\hline 3_45342613 & 3 & 45342613 & $\mathrm{~T}$ & $\mathrm{C}$ & C. micrantha & $\mathrm{C}$ & Ciclev10023477m.g \\
\hline 3_45711958 & 3 & 45711958 & $\mathrm{~T}$ & $\mathrm{G}$ & C. reticulata & $\mathrm{T}$ & Ciclev10018482m.g \\
\hline 3_50264450 & 3 & 50264450 & $\mathrm{C}$ & G & C. medica & $\mathrm{G}$ & Ciclev10021075m.g \\
\hline 3_50308586 & 3 & 50308586 & $\mathrm{~T}$ & $\mathrm{~A}$ & C.maxima & $\mathrm{A}$ & Ciclev10020530m.g \\
\hline 3_50881262 & 3 & 50881262 & G & $\mathrm{A}$ & C. ichangensis & $\mathrm{A}$ & Ciclev10020482m.g \\
\hline 4_128261 & 4 & 128261 & $\mathrm{~T}$ & $\mathrm{C}$ & C. micrantha & $\mathrm{C}$ & Ciclev10032342m.g \\
\hline 4_380914 & 4 & 380914 & $\mathrm{C}$ & $\mathrm{T}$ & C.maxima & $\mathrm{T}$ & Ciclev10033808m.g \\
\hline 4_390223 & 4 & 390223 & $\mathrm{~A}$ & $\mathrm{G}$ & C. medica & $\mathrm{G}$ & Ciclev10033662m.g \\
\hline 4_634314 & 4 & 634314 & $\mathrm{~T}$ & G & C. ichangensis & G & Ciclev10030516m.g \\
\hline 4_2154736 & 4 & 2154736 & A & G & C. reticulata & $\mathrm{A}$ & Ciclev10032524m.g \\
\hline 4_10567300 & 4 & 10567300 & G & $\mathrm{A}$ & C. reticulata & $\mathrm{G}$ & Ciclev10033209m.g \\
\hline 4_10730632 & 4 & 10730632 & G & $\mathrm{A}$ & C. micrantha & $\mathrm{A}$ & Ciclev10031169m.g \\
\hline 4_10887117 & 4 & 10887117 & $\mathrm{G}$ & $\mathrm{T}$ & C.maxima & $\mathrm{T}$ & Ciclev10033739m.g \\
\hline 4_12264521 & 4 & 12264521 & A & G & C. ichangensis & G & Ciclev10030907m.g \\
\hline 4_13917326 & 4 & 13917326 & G & $\mathrm{A}$ & C. medica & $\mathrm{A}$ & Ciclev10033976m.g \\
\hline 4_20183357 & 4 & 20183357 & $\mathrm{~T}$ & $\mathrm{C}$ & C. micrantha & $\mathrm{C}$ & Ciclev10033475m.g \\
\hline 4_22120298 & 4 & 22120298 & G & $\mathrm{A}$ & C.maxima & $\mathrm{A}$ & Ciclev10031235m.g \\
\hline 4_24151904 & 4 & 24151904 & $\mathrm{~A}$ & $\mathrm{G}$ & C. ichangensis & $\mathrm{G}$ & Ciclev10031585m.g \\
\hline 4_24484853 & 4 & 24484853 & G & $\mathrm{A}$ & C. medica & $\mathrm{A}$ & Ciclev10033431m.g \\
\hline 4_25206957 & 4 & 25206957 & $\mathrm{C}$ & $\mathrm{T}$ & C. reticulata & $\mathrm{C}$ & Ciclev10030476m.g \\
\hline 5_111138 & 5 & 111138 & $\mathrm{~T}$ & $\mathrm{C}$ & C.maxima & $\mathrm{C}$ & Ciclev10003884m.g \\
\hline 5_410426 & 5 & 410426 & $\mathrm{C}$ & $\mathrm{T}$ & C. medica & $\mathrm{T}$ & Ciclev10002260m.g \\
\hline 5_6729832 & 5 & 6729832 & G & $\mathrm{A}$ & C. reticulata & $\mathrm{G}$ & Ciclev10001528m.g \\
\hline 5_8523417 & 5 & 8523417 & G & $\mathrm{C}$ & C. ichangensis & $\mathrm{C}$ & Ciclev10000077m.g \\
\hline 5_20106308 & 5 & 20106308 & G & $\mathrm{A}$ & C. micrantha & $\mathrm{A}$ & Ciclev10000784m.g \\
\hline 5_21042485 & 5 & 21042485 & $\mathrm{C}$ & $\mathrm{T}$ & C. reticulata & $\mathrm{C}$ & Ciclev10003688m.g \\
\hline 5_21896647 & 5 & 21896647 & $\mathrm{~A}$ & $\mathrm{~T}$ & C. medica & $\mathrm{T}$ & Ciclev10003226m.g \\
\hline 5_24365312 & 5 & 24365312 & $\mathrm{C}$ & $\mathrm{T}$ & C. ichangensis & $\mathrm{T}$ & Ciclev10000255m.g \\
\hline 5_27123907 & 5 & 27123907 & G & $\mathrm{A}$ & C.maxima & G & Ciclev10003996m.g \\
\hline 5_33038626 & 5 & 33038626 & G & $\mathrm{T}$ & C. micrantha & $\mathrm{T}$ & Ciclev10001741m.g \\
\hline 5_40429150 & 5 & 40429150 & G & $\mathrm{C}$ & C. reticulata & $\mathrm{G}$ & Ciclev10001332m.g \\
\hline 5_41973357 & 5 & 41973357 & $\mathrm{~T}$ & $\mathrm{~A}$ & C.maxima & $\mathrm{A}$ & ET101382 / Ciclev10002683m.g \\
\hline 5_42898649 & 5 & 42898649 & $\mathrm{~T}$ & $\mathrm{C}$ & C. ichangensis & $\mathrm{C}$ & Ciclev10002424m.g \\
\hline 5_43054817 & 5 & 43054817 & $\mathrm{~A}$ & $\mathrm{G}$ & C. medica & $\mathrm{G}$ & Ciclev10000383m.g \\
\hline
\end{tabular}




\begin{tabular}{|c|c|c|c|c|c|c|c|}
\hline 5_43165502 & 5 & 43165502 & $\mathrm{~A}$ & $\mathrm{~T}$ & C. micrantha & $\mathrm{T}$ & Ciclev10003766m.g \\
\hline $6 \_353925$ & 6 & 353925 & $\mathrm{~A}$ & $\mathrm{G}$ & C. ichangensis & $\mathrm{G}$ & Ciclev10010953m.g \\
\hline 6_1942988 & 6 & 1942988 & $\mathrm{C}$ & $\mathrm{T}$ & C.maxima & $\mathrm{T}$ & Ciclev10010916m.g \\
\hline 6_1996772 & 6 & 1996772 & $\mathrm{~A}$ & $\mathrm{~T}$ & C. medica & $\mathrm{T}$ & Ciclev10013800m.g \\
\hline 6_7496346 & 6 & 7496346 & $\mathrm{~A}$ & $\mathrm{C}$ & C. micrantha & $\mathrm{C}$ & Ciclev10013603m.g \\
\hline 6_8942009 & 6 & 8942009 & $\mathrm{C}$ & G & C. reticulata & $\mathrm{C}$ & Ciclev10012181m.g \\
\hline 6_10155273 & 6 & 10155273 & $\mathrm{C}$ & $\mathrm{T}$ & C.maxima & $\mathrm{T}$ & Ciclev10013446m.g \\
\hline 6_11851009 & 6 & 11851009 & $\mathrm{~T}$ & G & C. ichangensis & $\mathrm{G}$ & Ciclev10012046m.g \\
\hline 6_13139033 & 6 & 13139033 & $\mathrm{C}$ & $\mathrm{A}$ & C. reticulata & $\mathrm{C}$ & Ciclev10011539m.g \\
\hline 6_13421556 & 6 & 13421556 & $\mathrm{C}$ & $\mathrm{A}$ & C. micrantha & $\mathrm{A}$ & Ciclev10013094m.g \\
\hline 6_13716315 & 6 & 13716315 & $\mathrm{~T}$ & $\mathrm{~A}$ & C. medica & $\mathrm{A}$ & Ciclev10011803m.g \\
\hline 6_22952731 & 6 & 22952731 & $\mathrm{C}$ & G & C. micrantha & $\mathrm{G}$ & Ciclev10010937m.g \\
\hline 6_23700512 & 6 & 23700512 & $\mathrm{C}$ & $\mathrm{A}$ & C.maxima & $\mathrm{C}$ & Ciclev10013459m.g \\
\hline 6_23938216 & 6 & 23938216 & G & A & C. ichangensis & A & Ciclev10010885m.g \\
\hline $6 \_25003350$ & 6 & 25003350 & $\mathrm{C}$ & $\mathrm{T}$ & C. reticulata & $\mathrm{T}$ & Ciclev10011556m.g \\
\hline 6_25003653 & 6 & 25003653 & $\mathrm{~T}$ & $\mathrm{~A}$ & C. medica & $\mathrm{A}$ & Ciclev10011556m.g \\
\hline 7_137886 & 7 & 137886 & $\mathrm{C}$ & G & C. medica & G & Ciclev10025242m.g \\
\hline 7_355705 & 7 & 355705 & $\mathrm{C}$ & G & C. ichangensis & G & Ciclev10027539m.g \\
\hline 7_2639448 & 7 & 2639448 & G & $\mathrm{A}$ & C. reticulata & $\mathrm{G}$ & ET079224 / Ciclev10024678m.g \\
\hline 7_2689161 & 7 & 2689161 & G & $\mathrm{A}$ & C.maxima & $\mathrm{A}$ & Ciclev10024810m.g \\
\hline 7_4057807 & 7 & 4057807 & G & $\mathrm{A}$ & C. micrantha & $\mathrm{A}$ & Ciclev10027450m.g \\
\hline 7_6084876 & 7 & 6084876 & $\mathrm{~A}$ & G & C. micrantha & $\mathrm{G}$ & Ciclev10027284m.g \\
\hline 7_8380800 & 7 & 8380800 & $\mathrm{~T}$ & $\mathrm{C}$ & C. reticulata & $\mathrm{T}$ & Ciclev10025881m.g \\
\hline 7_8928501 & 7 & 8928501 & $\mathrm{C}$ & $\mathrm{T}$ & C. medica & $\mathrm{T}$ & Ciclev10025300m.g \\
\hline 7_10698885 & 7 & 10698885 & $\mathrm{C}$ & $\mathrm{T}$ & C.maxima & $\mathrm{T}$ & Ciclev10026746m.g \\
\hline 7_13741299 & 7 & 13741299 & $\mathrm{C}$ & $\mathrm{T}$ & C. ichangensis & $\mathrm{T}$ & Ciclev10027319m.g \\
\hline 7_18363367 & 7 & 18363367 & G & $\mathrm{T}$ & C. reticulata & $\mathrm{G}$ & Ciclev10024790m.g \\
\hline 7_19114393 & 7 & 19114393 & G & $\mathrm{A}$ & C. medica & $\mathrm{A}$ & Ciclev10025197m.g \\
\hline 7_20103116 & 7 & 20103116 & G & $\mathrm{C}$ & C. micrantha & $\mathrm{C}$ & Ciclev10024684m.g \\
\hline 7_20899506 & 7 & 20899506 & $\mathrm{C}$ & $\mathrm{G}$ & C.maxima & $\mathrm{G}$ & Ciclev10025648m.g \\
\hline 7_21043581 & 7 & 21043581 & $\mathrm{~T}$ & $\mathrm{G}$ & C. ichangensis & $\mathrm{G}$ & Ciclev10026051m.g \\
\hline 8_44391 & 8 & 44391 & $\mathrm{C}$ & $\mathrm{T}$ & C. medica & $\mathrm{T}$ & Ciclev10028759m.g \\
\hline 8_756476 & 8 & 756476 & $\mathrm{~A}$ & $\mathrm{~T}$ & C. ichangensis & $\mathrm{T}$ & Ciclev10028168m.g \\
\hline 8_2438682 & 8 & 2438682 & $\mathrm{~T}$ & $\mathrm{~A}$ & C.maxima & $\mathrm{A}$ & Ciclev10028922m.g \\
\hline 8_3998031 & 8 & 3998031 & $\mathrm{~T}$ & G & C. micrantha & G & Ciclev10029698m.g \\
\hline 8_8442079 & 8 & 8442079 & $\mathrm{~A}$ & $\mathrm{G}$ & C.maxima & $\mathrm{G}$ & Ciclev10030161m.g \\
\hline 8_8518384 & 8 & 8518384 & A & G & C. reticulata & $\mathrm{A}$ & Ciclev10030164m.g \\
\hline 8_12939045 & 8 & 12939045 & $\mathrm{~T}$ & $\mathrm{~A}$ & C. micrantha & $\mathrm{A}$ & Ciclev10028114m.g \\
\hline 8_13486495 & 8 & 13486495 & $\mathrm{C}$ & G & C. ichangensis & $\mathrm{G}$ & Ciclev10029923m.g \\
\hline
\end{tabular}




\begin{tabular}{|c|c|c|c|c|c|c|c|}
\hline 8_14340205 & 8 & 14340205 & A & $\mathrm{G}$ & C. medica & G & Ciclev10028543m.g \\
\hline 8_17526645 & 8 & 17526645 & A & $\mathrm{T}$ & C. reticulata & $\mathrm{A}$ & Ciclev10029551m.g \\
\hline 8_23599778 & 8 & 23599778 & $\mathrm{C}$ & $\mathrm{G}$ & C.maxima & G & Ciclev10027940m.g \\
\hline 8_23989221 & 8 & 23989221 & A & G & C. ichangensis & G & Ciclev10029496m.g \\
\hline 8_24122489 & 8 & 24122489 & $\mathrm{C}$ & $\mathrm{G}$ & C. reticulata & $\mathrm{C}$ & Ciclev10027683m.g \\
\hline 8_24200344 & 8 & 24200344 & $\mathrm{~T}$ & G & C. micrantha & G & Ciclev10028652m.g \\
\hline 8_24991815 & 8 & 24991815 & G & $\mathrm{A}$ & C. medica & $\mathrm{A}$ & Ciclev10028485m.g \\
\hline 9_144478 & 9 & 144478 & $\mathrm{~T}$ & A & C. reticulata & $\mathrm{T}$ & ET080580 / Ciclev10005180m.g \\
\hline 9_177279 & 9 & 177279 & C & A & C. medica & A & Ciclev10005819m.g \\
\hline 9_231920 & 9 & 231920 & $\mathrm{C}$ & $\mathrm{T}$ & C.maxima & $\mathrm{T}$ & Ciclev10004227m.g \\
\hline 9_404236 & 9 & 404236 & G & $\mathrm{A}$ & C. ichangensis & A & Ciclev10004899m.g \\
\hline 9_1334064 & 9 & 1334064 & A & $\mathrm{T}$ & C. micrantha & $\mathrm{T}$ & Ciclev10004197m.g \\
\hline 9_13931363 & 9 & 13931363 & G & $\mathrm{T}$ & C. micrantha & $\mathrm{T}$ & Ciclev10006583m.g \\
\hline 9_14749302 & 9 & 14749302 & $\mathrm{C}$ & A & C. medica & A & Ciclev10006202m.g \\
\hline 9_16069488 & 9 & 16069488 & C & $\mathrm{A}$ & C.maxima & A & Ciclev10004121m.g \\
\hline 9_16250199 & 9 & 16250199 & G & $\mathrm{C}$ & C. ichangensis & $\mathrm{C}$ & Ciclev10004939m.g \\
\hline 9_18495356 & 9 & 18495356 & $\mathrm{~T}$ & A & C. reticulata & $\mathrm{T}$ & Ciclev10007029m.g \\
\hline 9_26923047 & 9 & 26923047 & $\mathrm{C}$ & A & C. ichangensis & $\mathrm{A}$ & Ciclev10004254m.g \\
\hline 9_29647599 & 9 & 29647599 & A & G & C.maxima & G & Ciclev10004997m.g \\
\hline 9_30507813 & 9 & 30507813 & $\mathrm{C}$ & $\mathrm{T}$ & C. medica & $\mathrm{T}$ & Ciclev10004179m.g \\
\hline 9_30666844 & 9 & 30666844 & C & $\mathrm{T}$ & C. reticulata & $\mathrm{C}$ & Ciclev10005358m.g \\
\hline 9_31143176 & 9 & 31143176 & $\mathrm{~A}$ & $\mathrm{G}$ & C. micrantha & $\mathrm{G}$ & Ciclev10006644m.g \\
\hline
\end{tabular}




\begin{tabular}{|c|c|c|}
\hline Marker ID & Flanking sequence & Publication \\
\hline 1_112523 & GAYGCTGCTACACCTACTCTAAGGCGCATTGAAAGGGCAGCCATAGCTGC[A/T]GCTACTCCTATCTCTTATCATTCCAGGTATTTCTTTYTTGTTYGAGAATA & Ahmed et al. (2019), Ann. Bot.123-7, 1231-1251 \\
\hline 1_429415 & CTATTGCCTTGGCCAATGGTGGTGGAAGGGATCCTGATTGGCAGGATTTT[A/G]TTGGTATCATTGTCTTGTTGGTAATCAACTCCACAATCAGCTTTATTGAA & New \\
\hline 1_1202115 & GGCAGCCATAGATCAKTTGTATCAGTTGATAAAAAATACCATGATTAATA[A/G]CCAAAATTCGGAAGTTGGTGGGCTAAATTTTTCAGAAATGGGGAGCTCTA & Ahmed et al. (2019), Ann. Bot.123-7, 1231-1251 \\
\hline 1_1445049 & СCTGTGACCTTGAAGGCCTGGCAGCATGGCCCAGTGGATCCCGAAACTGT[T/G]GCCGGTAAGATTGCCTCTGGAAGCATTGGGAGGCATGTAAAGGATGTAAG & Ahmed et al. (2019), Ann. Bot.123-7, 1231-1251 \\
\hline 1_4381520 & TTTATAACGTTTCAGATTTTGTGGGGAAGTCTTTAACCGCAGTATATGTA[G/C]CAAAAAGTATAAAAAAGGCAGCATGGGCTTGCACCGGCAGACTTGTGTTC & Curk et al. (2015), PlosOne 10 (5): e0125628 \\
\hline 1_10558128 & ACCAGGGCTTTGTATGTTGGCCCTAAAAAGAAAGGCTCCTTTCAGCACTC[T/C]AGTTTTCTATCCGGTGGCGCCACTACGGCTGCCGGAAGATTAGTTGCCCA & Ahmed et al. (2019), Ann. Bot.123-7, 1231-1251 \\
\hline 1_11872921 & ITTGAGATCGTGCAGCTCTAGTCTGGAGTTTGTGGAGGTAGATGTGATGC[C/T]TGCAAATGGTTGTGGGATTGAATTTTATTCTTTCTCCTACCTGATAYCAA & Ahmed et al. (2019), Ann. Bot.123-7, 1231-1251 \\
\hline 1_12808366 & TATTAACAATTAAAAATAATACWAAGACAATTTCATGCCTTCAGAGATCA[T/A]ATCTACTGCTTTTAGTTTTGTCATATTTGCTGCATCACGCATTTCTTCTG & Curk et al. (2015), PlosOne 10 (5): e0125628 \\
\hline 1_12909134 & TATTAAGTAAGAGATGGTATTGCAGATTCTGAAACGTAACTGCCAATTA[C/G]GGGAAGATTAATAGTCCCCAAAGACAACAAAAATTTTCAATGAGACTGC & New \\
\hline 1_14599773 & GCTCTGAAGCRGCTCGGGMWTCCCCCTCTTTAAGTTTTGCCATTGCCAAA[C/T]CGCGGTTAGCTTCTCTTCTCCTGAAAATTACCAAAGAYATAAAATTCTAC & Ahmed et al. (2019), Ann. Bot.123-7, 1231-1251 \\
\hline 1_23662492 & AATTTACACCTTGAAAGAAGGGATGTTGCTTGATCTCGGTTGCTCCTCGT[T/C]TGCAGGCTAATCTATTCTGGGGCTCTTTCACTAGTAAGCCCTTTATCAAA & New \\
\hline 1_24357413 & CACAAACATATAATATCAKTAATCAAAAAAGAAAAGAACCACACTGTCAA[A/C]AACTTCTAGCTATCCTCCTACAAMAGGTCAAAGCAAAGTTCATATCAGAA & Ahmed et al. (2019), Ann. Bot.123-7, 1231-1251 \\
\hline 1_25122651 & GCGAGCTATTTATAGAGCAGGATGGTGACTAATTTCCTTCATTAACATAT[G/A]GCTCTTAGTTAATTARGTGARTATTGGTAGGYTCAATGGTAGTCCACGYA & Ollitrault et al. (2012), BMC Genomics 13:593 \\
\hline 1_25274313 & ATGATAGCATGCATTATAGAATCACCTACATAAAAAGATCAGCAGCCATC[A/T]GCAAATGAAAACTAGATTTCTGATCCTTTATAACAGAAGAGTCGTGCTCT & Ahmed et al. (2020),Front. Plant Sci., fpls.2020.00939 \\
\hline 1_27714027 & CTTGACCTCAACAATKTRTTGGCCTCTTTTGACAACAACAGGCTCATTAG[A/C]AAGAACATTTTCCAAGTGGTCAAGAAGCTCTTTAGCCTGGCATGAGCCAA & Garcia-Lor et al. (2013), APS 1(4):1200406 \\
\hline 2_148798 & TCAAGTGTGCTTTCAGCTTCTACACATATTTGGGCCATGGTACGGACTGC[G/A]ACCTCCGGATAAGCTCCAGCTGCAGTTTCACCACTTAACATCACACAGTC & New \\
\hline 2_411531 & ACAAGATTCTGACAATGGCAGCATAAGTGTGAACATTATGCCTAAACCCT[T/C]TCGCTTTTAACAGTTCAAAGAATGTTAATGCAGCTCCGGGGTCTTTTCTG & Ahmed et al. (2019), Ann. Bot.123-7, 1231-1251 \\
\hline 2_7448065 & AGATGCAAATTTCTGTTTAATATCAGAAGTGCTAGATGTCATGGAATTAT[C/G]TCCAGGTGCTGGCAATTTCCCAGCTATTTCAGACTSRTTTGAGAATTCAG & Ahmed et al. (2019), Ann. Bot.123-7, 1231-1251 \\
\hline 2_7659486 & AATGGTGTGAAGATGTCATGGCTGATGAAATTAGAACCCTGCAGCGCCTT[G/C]AACGATTGCAAGCTACCAGTGACAATCTTCCAAAGGAAAAGGTTGCTTCT & Ahmed et al. (2020),Front. Plant Sci., fpls.2020.00939 \\
\hline 2_8056523 & TTTCTTAAGAGTTTCCACCYCGGACCATAATCAGCAAAGACCATGTCTTG[G/A]GCGTCGTAGGCCAAGYGGGTTGCACCGGCGTTCGGCGGCCGGTTCGAGAA & Garcia-Lor et al. (2013), APS 1(4):1200406 \\
\hline 2_11091624 & GGATATTCACTTAAAGCTGACTTAAAGAGCAATGAATTYCTTCAAACCTA[G/A]TGGAATATTCTATGCCTTCTCAGTTCTTAAATGCACTTTCCAATAAATAA & Garcia-Lor et al. (2013), APS 1(4):1200406 \\
\hline 2_11492295 & ACAAAAGATTACAAAGAAAATGAAAAAAGTTAAAAGAACAAACCTGAAA[C/T]TAATTGAAGAAGGTGAAATGAATGGCTTGAGGAACAGAGTTTGAGGCATT & New \\
\hline 2_15483255 & ITCCAAGACCAGGCTGGCCATACTATGAAGGTATTGCACAGCGTAAACAA[G/C]TCGAAGTTCRCCATTTTGATCTTCTTCCAGAAAGGAATTGGGAGGTTGAT & Ahmed et al. (2019), Ann. Bot.123-7, 1231-1251 \\
\hline 2_21022776 & ATTGAGAMACGAAAAACTCAAATGAGCACGAAAGCGAAGGACCCTTATGT[G/A]GGAATGCTTGTCACGAATGGACAACACCTGGGAAATCAAATCCATTTCTT & Curk et al. (2015), PlosOne 10 (5): e0125628 \\
\hline 2_25198777 & ATTGGAGATGATGGCAGTTTGTGGGTTTGGGGAAAGTCAAAACGTGGGCA[A/G]CTTGGTCTCGGGAAAGATATCAYAGMGGCCATTGTACCCTCAAGAGTCGA & Curk et al. (2015), PlosOne 10 (5): e0125628 \\
\hline 2_26306845 & AGATTTTGGATGTAATCAATCAAATGGTTTAGTACTGAGTAAGTTACTTG[T/C]CTGGTTTTGACAACATGCAAAACCTGAAAAAGTCCAGTGGGTAAACATAT & Garcia-Lor et al. (2013), APS 1(4):1200406 \\
\hline 2_28936300 & AATTATGCTTTGGCATAATGCCATAAAAAGAGAGTTGAATGATATAGCTG[C/A]GGCAGCTAGAAAGATACAACTTTCTGGAGATTTTTCTGACTTGTCRGCAT & New \\
\hline 2_31567713 & CCCATGAYGAAAACATTAACAGCAATGCAAGCAGATGGCAGCCAAGGAAG[T/C]AATGGTACTCCCCACATCTTAGGCTTTCTTGCTTCCTTCASRGTCAATTG & Ahmed et al. (2020),Front. Plant Sci., fpls.2020.00939 \\
\hline 2_31581849 & ACAATCAAAAGCCTTGGCACATTCTCTACTAACAAATCCATGTATTCCAT[A/G]TAGCAGTAAGCTGGGTGGTTGATCGGAGGYGGCGGTARACTAACAAGCAC & Garcia-Lor et al. (2013), APS 1(4):1200406 \\
\hline 2_35686712 & TTGCCGGCGGCGGAGCTAGCGGGGCCATCGACGATTTTGAAAGGGTTTTT[T/G]AGAACAGAAGGGAGAGGSAGTGCGTGATCGATTCGACTCGTTTGGAGTTC & Garcia-Lor et al. (2013), APS 1(4):1200406 \\
\hline 3_105647 & TAACATTTCCAGTAGTGACACAAGAAACAAGTGCTTGCACTATTCTCTCC[G/A]GCCAACGTGAATCTCCCAAAGATGCTGGATGGCTTGAACTTGTATATTTA & New \\
\hline 3_165889 & GTCCGCTGCTGTAGCTGCAKCGGGTAAGGTYGATCATCATCTTATCGTGG[G/A]TCATCAAGTTGTTGCAGCAGCTGGTCATGCTTATAATGTTGGCCTCCAGT & Curk et al. (2015), PlosOne 10 (5): e0125628 \\
\hline 3_2073865 & CTAGTTCAAGGAAAAGAKTACAGAAAAGAAAGAATTCAAACTAGTGCTCG[T/C]ACATTGGATGGARGAAAGCAGCTGCAACAACCAATATTCAAGGTCGACAA & Curk et al. (2015), PlosOne 10 (5): e0125628 \\
\hline 3_5866407 & TGCGTGGCGGATGGGAATGAGGTTATGAGGTTCTACTGCCTCGGCCCCAC[G/C]GCTGCCAGTGGAGCGTGCGGCGGAGGKTACGGCGCGTGGGGGTTTCCTGG & Ahmed et al. (2019), Ann. Bot.123-7, 1231-1251 \\
\hline 3_11355960 & AACGTTTTGGATGGTTTCKSTTGGATTTGGATTCAACCAAGACACCAATG[G/A]TTATGTATTGGTGAGGATTGTCAACTTTCAGGCTCGTTAYGATGCGATTG & Curk et al. (2015), PlosOne 10 (5): e0125628 \\
\hline 3_14861567 & GGCAGAGAAGGCRCGTATTTATTAAACACAATTCTATTAGGATTGAGAAA[A/C]ATGTTATATGCTGTGCTAACATCCGACAATTATGCCTTGAAATATTAGCT & Ahmed et al. (2020),Front. Plant Sci., fpls.2020.00939 \\
\hline 3_24894898 & ACAGCAGCTGTGAAGAGACTAGTGACTGCTGGCTCAATGAGGAGAATACA[C/T]GAACGTGTACTAGGGCCGAGCAGGACTGGAATTTCTAGCTCAACCAGTGA & Ahmed et al. (2019), Ann. Bot.123-7, 1231-1251 \\
\hline 3_27184394 & AAAATCTACCAGAAATACAGGCAGCAAAGAACGAATATGGGAAAGCAACA[G/A]GGAAAAATATTTTGAGACTGCTGTTGCTTTAGTTTTTGGCCAGGGGGAAG & Ahmed et al. (2019), Ann. Bot.123-7, 1231-1251 \\
\hline
\end{tabular}




\begin{tabular}{|c|c|c|}
\hline 3_29352884 & TTCTTGTCCAAAATCCCAAATCTTGACACCTTGTTCTTGTCATAAATCAC[A/C]GGGGAGCCACCTCGGATCATCTCWTTCAACTTGAACACCACTTGCTGGTC & Garcia-Lor et al. (2013), APS 1(4):1200406 \\
\hline 3_29381066 & CGTCAGCAGAATCCTGTTGGATATACTGTACCCATCCAGCTTCCTGTTAT[T/C]AATCAAGTATTCAGTTCCAATCATGCGGTAAAAATATCACCAAATTGTCC & New \\
\hline 3_45342613 & AGTTGCAACTGTAAGCAATGTGAAAGTTGTGGAGAGCTCAAAGAGAGTAA[C/T]GTTCAAATCCAGTGATGTTATGCTACGTATTCTGGGATTCTCCCTCACAT & Ahmed et al. (2020),Front. Plant Sci., fpls.2020.00939 \\
\hline 3_45711958 & СTTTTCCTTGAATTTTCAGTTTGATTTAATGGAAGTCATATGTATCTTTT[G/T]AGAAGCTAAAACATGCCAAAATGTTGAACTTTGYAGTGGCCGATAGTGGT & Garcia-Lor et al. (2013), APS 1(4):1200406 \\
\hline 3_50264450 & ATATGATCACAAGCAGAACTTGCAGCAGAAAGTGCACTTGATAGCTTCCT[G/C]GCTTTGATGATGGCCGCACCTCGCTGCTGCACGGTGGTRATGAACTCGGT & Ahmed et al. (2019), Ann. Bot.123-7, 1231-1251 \\
\hline 3_50308586 & CGTGTTGCTAAAGACATAGCTGAAAACAACCCTGGAAGCCGCGTTTTGCT[A/T]ACCACTTCTGAAACTACCATACTTGGGTTTCGCCCACCAAACAAGTCCCG & Garcia-Lor et al. (2013), APS 1(4):1200406 \\
\hline 3_50881262 & CACTCGAAGGACACAACCAACCCTCGATGCCGAACCTGTTTATAGTGCTA[A/G]ATAGTGCTGTCTGTGATCATGTAACCTTTGAAGCATTATTATTATATT & New \\
\hline 4_128261 & GTGCAATTTGTGGATCCTTATGAAGCTGCTGAAGCTCAACACCATATGAA[C/T]GGGAAACGTTTTGCTGGGAGGGAAATATCTGTAGTCCTTGCTGCAGAGWC & Ahmed et al. (2020),Front. Plant Sci., fpls.2020.00939 \\
\hline 4_380914 & CCAATAGCAATTGTATCTTCAAAATAATTGACAGCAGAAAAACTACCAAC[T/C]GAAATTGAGATTATATCAACACCATCAGCAATTGCATCATCAAATGCTGC & Ahmed et al. (2019), Ann. Bot.123-7, 1231-1251 \\
\hline 4_390223 & KATYAATAGTGCTTGCAGCCACAGAAATAAACCAAGGTGCAACATTTGTA[G/A]TAGTTGAACGGGAAGGGCCGTCATTACCAGCCGAAGCCGAGGYCAATATT & Ahmed et al. (2019), Ann. Bot.123-7, 1231-1251 \\
\hline 4_634314 & GGGCAGAATGATGCAGATCTTGATTTTAATGCTGTACAGCGTGGGGATGC[G/T]GAGATGGCACAAAAGTTGTATCGTGTAGTTCGTGCCTGCATTGAGATGGG & New \\
\hline 4_2154736 & ATTTCATAGCATGAACCACAGCTTAGGCCATTGTTGAATAGAGCGGTACT[G/A]AGTGCTGCAGTGTTAGTCCCRTAGCCTTGGCTGTACAAATTGCCATACCC & Curk et al. (2015), PlosOne 10 (5): e0125628 \\
\hline 4_10567300 & AACTTCTCCATTTTTACTTGTTTTAAATACTTTCATGTGTTATATRCTTT[A/G]GTAACTGCAATTCACCATTATGCTAAATGGCTTGGATTGTCTATTATTGM & Curk et al. (2015), PlosOne 10 (5): e0125628 \\
\hline 4_10730632 & AAGCCGAACGGAAAGTGCTGAGGCCACAGTTGAGTAAAATTGCAATAACA[A/G]GTCTTGAGTTCTCAGAAGCACTTCCATTTGCTGCTTTTGCTTCTTTGCTA & Ahmed et al. (2020),Front. Plant Sci., fpls.2020.00939 \\
\hline 4_10887117 & AGCTCAGACAGCCCTTGAAGAGTGGATGTGGACTTTGGCCAAAATTGTTC[T/G]GCGTGAAGAAGTGTTGCCGCAGTTATTTTTGGCTGAAACAGGCACTGGAA & Ahmed et al. (2019), Ann. Bot.123-7, 1231-1251 \\
\hline 4_12264521 & TTAATAGTCGCCAACTCCTGGACGTTCATGATGAAACTCCCATCACCATC[G/A]ATATCTACAATGATGGCATCAGGATTTGCAACAGCAGCTCCGATTGCAGC & New \\
\hline 4_13917326 & ACAGCCACCRTGGAATGGAAATCACAGGTGAATGTATCTAAAGCATTGGA[A/G]CGTCGCAATCCTGAGAAACTACTAATTCTWGAAGCAGAYGGCTGCATATA & Ahmed et al. (2019), Ann. Bot.123-7, 1231-1251 \\
\hline 4_20183357 & CATTCTTAGCATGTCCCATCTCGACACTCGGTGAGTTTGAGTATGTTTTC[C/T]GACCAGCGATCTYAGAGCAATAYCTCTTGTAATAACTCATGGCGAATGAA & Curk et al. (2015), PlosOne 10 (5): e0125628 \\
\hline 4_22120298 & CCCACAAGTGCGCACTCRAGCAGGCCCTCAAGTGCCCAATCGAGCAGGTC[A/G]GAGAGTCAGGGGCTGCAGGGATTGGAGAATGTGGTGAAGCCGAGTAGGCC & Ahmed et al. (2019), Ann. Bot.123-7, 1231-1251 \\
\hline 4_24151904 & ATGACATTTGGTTCATTGTGAAGATAAGCCATGCCTCTGGCCACAACAGA[G/A]TTACAGATATTAGCATAATATTGAAATCCACAAGGGACTTTTATTCTATA & New \\
\hline 4_24484853 & TCGATTGGGACGCGATCGTGCGCGGCAGACACAGAGCAGCTACCGTGCGC[A/G]TCCCAATCGCACTCTTGACAGAGCACCAAATTGTCAGTGGCGCACCGGAA & Ahmed et al. (2019), Ann. Bot.123-7, 1231-1251 \\
\hline 4_25206957 & CATGGCGGTGCAGAATATAGAGGTACAGGTCGTGGCTTTGTAGTGTTCCA[T/C]GCCAAGTTTGCTGAAAATTATCGGCTTTACTCCCGCAGCCACTTTGTCAA & Ahmed et al. (2019), Ann. Bot.123-7, 1231-1251 \\
\hline 5_111138 & TTTGAAAACAATTGATGCWKCAGCCATTTCAGCAGCAAGATCCGAGCTAC[C/T]GGTACATTCTTGAACCGGACGGTCATAATCAATAYCCTCAGGRCGAATCC & Ahmed et al. (2019), Ann. Bot.123-7, 1231-1251 \\
\hline 5_410426 & GATGAACAGGGYGCCACCTGTGGAGCATGCGGGGATAACTATGGCACTGA[T/C]GAATTCTGGATCTGCTGCGATATCTGTGAGAAATGGTTCCACGGCAAATG & Ahmed et al. (2019), Ann. Bot.123-7, 1231-1251 \\
\hline 5_6729832 & TCACTTGATGAGCGCCGATGACCTCCTCTGCGTACAGGTGTCTCAGGCTC[A/G]TTAAGGAGGTCATCGAGCCAAGAAGGCTGCTCCTCCATTAGGAAGCTCTC & Ahmed et al. (2019), Ann. Bot.123-7, 1231-1251 \\
\hline 5_8523417 & AAATTCTTAGAGAACTTAACTCTGATTATTGCAAATACAAGCTGTTATAT[C/G]TCACTCCAGAGAAAGTTGCAAAGTAAGTGTTACCTATATCAATTATGATG & New \\
\hline 5_20106308 & GCTTCTTTTAATRGCTTTACCTGTTCTATTCATAGTCGTTTTGATTTGCA[A/G]AGATAAAGGGTTCAATCCTATGGTCGCTGCKGGTAAGTTCATTTGCTCGT & Ahmed et al. (2020),Front. Plant Sci., fpls.2020.00939 \\
\hline 5_21042485 & AGGTCTCAAGGATTACCTGAAATKTCACTAGAGGAAGCATTCAAAATGTA[T/C]TGTGATGGTGTCATTGGGTTCGGTCCATTTTGGGAGCATATGTTAGGGTA & Curk et al. (2015), PlosOne 10 (5): e0125628 \\
\hline 5_21896647 & СATGTATGTCGGCTTCTCTGCTTCAACTGGTTTGCTTACAGCCTTCCATA[T/A]TGTGCATGGCTGGAGCTTTAAGATCGGTGGGACTGCACGWGAACTGGATG & Ahmed et al. (2019), Ann. Bot.123-7, 1231-1251 \\
\hline 5_24365312 & CTGTGGTTATAGAAACAGGAGGCCGATCATTCATTGCAGAGAGCCATGTC[T/C]GGAACAGCCACGTAAAGGAAGCCTCTGACTCATCTAGAAGTAAAGCACAA & New \\
\hline 5_27123907 & AGAGCAGCGGTGATTTGTTGCACAGTGCTGCAAAGATGGCTGTTGATTGC[A/G]TTAACGCTAGAGAGATTGAGCTTGGCAGATGGGATTGTGCTGCCAAGAAC & Ahmed et al. (2019), Ann. Bot.123-7, 1231-1251 \\
\hline 5_33038626 & ATTCTCRTCCAATAAGACATTGGAGGCTTTGATACATCTAAAAACAATGG[T/G]TCTGTCAAATCCAACRTGAAGATATGCAACTGCATTAGCTATTTCCATGG & Curk et al. (2015), PlosOne 10 (5): e0125628 \\
\hline 5_40429150 & AACGAAGACACGAAGAATACGAGTTCCCGCMCAACCGATGGCTCCGGTTC[C/G]GGTCGCAGAAAAGGAYCGGTTTCCGCTCACGAGTTGCATTATACTAAGAA & Ahmed et al. (2019), Ann. Bot.123-7, 1231-1251 \\
\hline 5_41973357 & AATTCAGCATAYAAGTTCATGAAATTACRCGAAGCTTACGAAACTCTATC[A/T]GATCCCAAGAAGCGTGCGGACTACGATCGTACCCTTTTCAGGCGAAGAAK & Ollitrault et al. (2012), BMC Genomics $13: 593$ \\
\hline 5_42898649 & GCAAAGCAAGCTGTTGTCTCAATACCCATTTCAAGAAGAGCAGCAGCAAT[C/T]CTGATATCTTCACTCCCATTTAGCGTCATTTCACTACCCAAATGCTCAGA & New \\
\hline 5_43054817 & TATGTGGGTAGAAAACTGACCACAGAGCAAGAGTGATGCTGTTGGCATCC[G/A]CTAAACAGTGAATYYTGTGTGACCCCAGGACCATACTTCTTTGCAGCAGC & Ahmed et al. (2019), Ann. Bot.123-7, 1231-1251 \\
\hline 5_43165502 & GCTTAGTATTAAGAACATGAACATATGCTATTTCCATGTTCACAATCATT[T/A]ACATGTGGTCTTGTTTGGTSCAGGCAGGGAACGCATTGGATGCTGTACGA & Ahmed et al. (2020), Front. Plant Sci., fpls.2020.00939 \\
\hline 6_353925 & GTTTTAATCGCCCCTACCACTTCACCTATGTGCCTTGGATCCTTAGATCG[G/A]AAAGATACCTCCTTCAGATATATTGCCCCTTGGTGTTTCAATGAGTTTGT & New \\
\hline 6_1942988 & ATGGAAGCTGCGCATTCTAAGGCCACACAACTGGCTGGGGAAGCCATTGG[T/C]AATGTGAGGACTGTTGCTGCCTTCAATTCAGAGTTAATGATTGTTGGTCT & Ahmed et al. (2019), Ann. Bot.123-7, 1231-1251 \\
\hline
\end{tabular}




\begin{tabular}{|c|c|c|}
\hline 6_1996772 & TATGTTATCCTGTCCAATACATATGCAGCTGCTCGGAGATGGAAAGATGC[T/A]GCAAGTTTAAGAGTCTTTATGAGAAACAAGGGCATGAAAAAGACACCAGC & Ahmed et al. (2019), Ann. Bot.123-7, 1231-1251 \\
\hline 6_7496346 & AATTTTTGATACCCTTGTTTCCAGGGAACAAATCCTGAGAAGCTGTTGTA[C/A]GACAAGTTCAAAAGAGTCAACTCTTGAAGACTTGAAATGTTTCCGGGTAT & Curk et al. (2015), PlosOne 10 (5): e0125628 \\
\hline 6_8942009 & CAAAATCTTTGYCTTTTTGGTTTGTCCTGCAGCCTGTGCTTGCTGTAAT[G/C]TATCTGGTGCAATACATGCCGACCATTGGATACTGGCTCATGGACAAGGT & Ahmed et al. (2019), Ann. Bot.123-7, 1231-1251 \\
\hline 6_10155273 & CAATATAACATGTCTATCCATTGGTATGCATCAATTATGCAATCACAAGT[T/C]TTGCATGCATTACTAAACACTGAGCAAGGCTGCCGGTCTTCCTTTTGRAT & Ahmed et al. (2019), Ann. Bot.123-7, 1231-1251 \\
\hline 6_11851009 & AATAAACATACTGCCACTCTAAAAGGAAGGCAGCTTAGTTTCAGAAAATT[G/T]CATCACCTGATTCTCAATCTCCTCCTCAATGGTTATTTTCTCTGCTTCTT & New \\
\hline 6_13139033 & CAACCCTCTGATCGGATTGCAGATATGGAAATACACCCTTATGAGCCGCC[A/C]CACTGGTTGAATGACTTTAGTGGAGTGATGAAAAATGTTTACGGGCCTTC & Ahmed et al. (2019), Ann. Bot.123-7, 1231-1251 \\
\hline 6_13421556 & TGGTCCCAATCATCCCAAGAACTTGAGCCATAAGAATTAYGATGCCCAGC[A/C]GAGTACTGCCCACCACCAGCAGGAGAATCCYRACCTTTGTTACCATGGTT & Curk et al. (2015), PlosOne 10 (5): e0125628 \\
\hline 6_13716315 & TCATGTTTGCTAGTCTAGCCTTTATGCCTTCAGTACAACTATGGACAATA[A/T]TAAGCGCAGCATGGTTCCTCACTTTCAGGTCTGATGTTCAAGGAAATCRT & Ahmed et al. (2019), Ann. Bot.123-7, 1231-1251 \\
\hline 6_22952731 & GAACATCATACAATGGTATTCAATATCTTATGGGAAATGGTTGTCAGCTC[G/C]AACATAGATATGAAAATCAATGCTGCCAATCTGTTGAAAGTCATTGTAAG & Ahmed et al. (2020),Front. Plant Sci., fpls.2020.00939 \\
\hline 6_23700512 & AGAGGGCATTTTGGGGAATGACATGTAAGGATGTTGTTTCATGGAACGCA[A/C]TAATAGATGGATTTGCTTTGAACGGAAAATTTGAAGAAGCATTTGATCTT & Ahmed et al. (2019), Ann. Bot.123-7, 1231-1251 \\
\hline 6_23938216 & GTGGTTGCTCAGCCAATTTTGGAAGGTTTAGAAGATGTAGAGGAGGAGGA[A/G]GATGGATTGGCATGTATGGTGTGCCGAGAGGGCTACAGTTTGAGGCCTAC & New \\
\hline 6_25003350 & CCGAATGGGGTCCACCATCCTGCGTTGAAAACTAATGGTACTGTACTTAC[T/C]TTTGGCTCAGATGCACCCCTTTGTGAATCAATGGCATCAGTTCTGAATAT & Garcia-Lor et al. (2013), APS 1(4):1200406 \\
\hline 6_25003653 & CATGTGGCTTGCTTTCCTGGGGCTCCGTGGCCATACCCATGGAATTCGGC[A/T]CAATGGAGCCCTCCAGTTACCCCACCTGCGATCCTTCCTCCAGGCTTCCC & Garcia-Lor et al. (2013), APS 1(4):1200406 \\
\hline 7_137886 & GCAGCATGGTGTCTGCAACCTTTGAAGGAATACATATTTTGGAAATATAG[G/C]TCTGCCCAAAAGTGAGTATGAGTGAAATGAACCCCAGAACCATAAGCTCT & Ahmed et al. (2019), Ann. Bot.123-7, 1231-1251 \\
\hline 7_355705 & TTTGAGAGGATGTTGAATATTGATGTCGAAACACCCGCTGATGCCAGCTC[G/C]AAGGGACCTTTTCAGTACATTAATTATATAAGAGCTCAAGGGGGAATAAA & New \\
\hline 7_2639448 & ACAAACTCTGGAACCCCATCAATAAAAGCAACAGCACAGTCATTCTTCTT[A/G]TTCACAAGATAAAAGGTATCTTTAACCAACTGATTTTGAGCTTCCCAGTC & Ollitrault et al. (2012), BMC Genomics $13: 593$ \\
\hline 7_2689161 & YGAAACTGATARAACAGCAGCATCAAACGATAACACATGGTGRTCATCAG[A/G]AGCGCCAAGGTTTGCCTCTTTGTCATCAAAAATTGGGCTGCCATATATYT & Ahmed et al. (2019), Ann. Bot.123-7, 1231-1251 \\
\hline 7_4057807 & TAGCCTCCTCTAGCTGCTGACCAATTTTCCTCATCAACTCTGTCCTTTCC[A/G]TTTCTCCACATTCCAACATAATTGCTGCCCTTAACAACTTAAGCAAGAAA & Ahmed et al. (2020),Front. Plant Sci., fpls.2020.00939 \\
\hline 7_6084876 & ACGTGCGACAAAGAAAGAAAWCTTCATCCACAGGAAATAATTGAATTTCC[G/A]TCCGTGGTGGTGAGTGGTGTAAGCGGAGAGATTATAGCATGTTTTCAAAC & Curk et al. (2015), PlosOne 10 (5): e0125628 \\
\hline 7_8380800 & ACATAAGGCAGCTCCTCGTAACCAAAAGCCTCAACGACGTCGTTGAATTC[C/T]TTGGCTGTCYTCACATCATCAAATCCCCTGAAAAGAACTGATCCTGCTTT & Ahmed et al. (2019), Ann. Bot.123-7, 1231-1251 \\
\hline 7_8928501 & ATATGTTTCAGCTGCAKTTGCTTATGTACTGRTGTTCATTTTACATGACA[T/C]ACAAATATTCTCAGGGATCCGATTATGAAGAATTTGTAAAAACAGCAGCT & Ahmed et al. (2019), Ann. Bot.123-7, 1231-1251 \\
\hline 7_10698885 & AAATCCTTGCTAGCATTCCCTGCCAACTCCAACACTTCGGCAGTTAGATA[T/C]TCCAAAATGGCTGCTGTATAAACAGCAGCTGTTGCRCCAACCCTTCCGTG & Ahmed et al. (2019), Ann. Bot.123-7, 1231-1251 \\
\hline 7_13741299 & CAATAGTAGGAATTCCAATTCCATTCACTGAAATGGAAACCAAATTTGGA[T/C]TATTCCGCATAGCAAAACTAATGCCACTTTGAGTAATAAAATCACAATCC & New \\
\hline 7_18363367 & TCATTCTTCWTGACTTACAGTATATGGAGCCACATGAACATGAAAAGCTT[T/G]CCCAAGTTGAGCTTCTAGTGATTGATGAAGCTGCAGCTATTCCCTTGCCT & Ahmed et al. (2019), Ann. Bot.123-7, 1231-1251 \\
\hline 7_19114393 & AGAGTGCCTCGACTCGAACGAGTTACAAGTGGCTCAGATGATATTGGCCC[A/G]CCTCAATCACAGTCAAAACCTAGTAGGTTCACCACCAGTAGTAGTWGCTG & Ahmed et al. (2019), Ann. Bot.123-7, 1231-1251 \\
\hline 7_20103116 & TTTCTGCTAGCATGCATGATGGAGGAGGATGTGTCTGTTTGCTCCAGGGT[C/G]TTAGCTGCTGATGCTACGAAGCAACTTCTCAAGCTGTTAGGATCTGGTAA & Ahmed et al. (2020),Front. Plant Sci., fpls.2020.00939 \\
\hline 7_20899506 & CACGAATTTTGAGCGGTTTGTTGCTGGTGCTGCAGCTGGAATAACTGCCA[G/C]CTTGCTTTGCTTACCATTGGACACTGTATGTGCCATTTTAGAATTATTT & Ahmed et al. (2019), Ann. Bot.123-7, 1231-1251 \\
\hline 7_21043581 & AAAAGCTCGTCCCACACGAACACTTCCACCTGCTTTCCCCGCAGTTCTTG[G/T]AATGACTTCTCAGATCCGATACAACCGAAAAACTCTTCTTGTGGCACTTA & New \\
\hline 8_44391 & CCCYTTTCCCTGCATAAACCATCAACTAGTCYAACAAAWGTAGTCACATT[T/C]GGAGAATGACCAGCTTCTARCATTTCAATGCAGTACTCGACRGCTTCTTC & Curk et al. (2015), PlosOne 10 (5): e0125628 \\
\hline 8_756476 & AAATTACGAGAATATGAAGAAATATGACAGTACCAAATCAATGAGACGGG[T/A]TAAGTATCCTCCCAAGTCCTCTGTTTCTTCTTTTTTACCCACAGGCAAAG & New \\
\hline 8_2438682 & AAAAGTTTTTTAYTTAGTTAATTAACTATTGATAAAATTTGAGTGGTGTT[A/T]GTTGGTTATGGGGAATTGAAATGAAAATAATCATRTGCAGACAGATACAG & Ahmed et al. (2019), Ann. Bot.123-7, 1231-1251 \\
\hline 8_3998031 & CAGGCATTGTAGGTAAGAGGAAGTTAAYATGYCGATACCAGTACCATGTT[G/T]TGTGCAGCAGTGTGTTTACAAATTAATCTCATTCATCWCTAGCTTTCAAA & Curk et al. (2015), PlosOne 10 (5): e0125628 \\
\hline 8_8442079 & GTGGGGCAGCACGCCCAAGACCAGCTGGACGAGCAGGAGGGTTAACAGGC[G/A]TTGTAGATTTTGCAGCTGAAGAAGCAGGCTGTGGTRAARGTTGTACGTTA & Ahmed et al. (2019), Ann. Bot.123-7, 1231-1251 \\
\hline 8_8518384 & GTGCTAGTACCGGTGGGTCCATGCTTATTATTGGCTCTGCTGCTGGAGTT[G/A]CATTTATGGGAATGGAAAAGGTGGACTTCTTTTGGTATTTCCGGAAGGTA & Ahmed et al. (2019), Ann. Bot.123-7, 1231-1251 \\
\hline 8_12939045 & AAGCAAATCGCACCTCCATAGTACACAGCAGCTTGACCACTGCTATAATG[A/T]GTTATTGCACCGAACAGATTAGTATTGTAAGCTAAAGCCAGTGCAGCCAG & Ahmed et al. (2020),Front. Plant Sci., fpls.2020.00939 \\
\hline 8_13486495 & ATACCAAATGCTTTGGAAACTCCATCATCAACTCTGAGACATGAATTGGT[G/C]TCTCGTATATCTTGACATGCCCATCTGAGTTTACAATCTTGATTGTTCCG & New \\
\hline 8_14340205 & ACTGCAGCAGGTCACACGGTATCTTGAAAATTTCGAAAGATATGGAGAAG[G/A]AGGAAGACAAAACGCAGCGTATAGTGGAGAACTCATTRTTTGCAACGATG & Ahmed et al. (2019), Ann. Bot.123-7, 1231-1251 \\
\hline 8_17526645 & AGCTACAATTTCCCCACTTGAATCTCTGACCACTGCTCCAAGTCCTCCCM[T/A]TTGCTTTGATAAATTTGTAGCCGCATCTACATTTACTTTAAACYAACSAG & Ahmed et al. (2019), Ann. Bot.123-7, 1231-1251 \\
\hline 8_23599778 & GACAGCAGTGACTTCTCCAATGCTGTTCTCAAATACATAAGTGAGATTCT[G/C]ATGGAAGAGGACTTGGAAGGGAAGACCTGCATGTTACAGGACTGCTTGGY & Ahmed et al. (2019), Ann. Bot.123-7, 1231-1251 \\
\hline
\end{tabular}




\begin{tabular}{|c|c|c|}
\hline 8_23989221 & AACAGCAGAAACCCTAGAGCCTGGCCAGCAACAGCCGCAAGAAAAATTCC[G/A]GCGTTGAACGACATGAGAGAAAGCATCAGCAGATATCCAAGACTAACCCT & New \\
\hline 8_24122489 & AGGTGCAGCAGGCCTTTGTTGAAGAGTAGAGGATCCATAAAGAGATGGAG[G/C]GGACTGAACAAAACCTCCAGCTTGCGCCTGACCAGCTGGAGGAACAGYTC & Ahmed et al. (2019), Ann. Bot.123-7, 1231-1251 \\
\hline 8_24200344 & TTTCGACTTTGAAGTTTTTGTGGCAGCAGGTTAGCTGTGTGTAGCAGCTC[G/T]TCACCAAACTCCAAAAGTCATACAACTTAACAAACAAACAAATCAAATAC & Ahmed et al. (2020),Front. Plant Sci., fpls.2020.00939 \\
\hline 8_24991815 & GTTGAGCAACTTGYCAAAGTGAACCCTGTGCACTTGAGCAGCAATGAGAA[A/G]CTTGCTTTCTGGATAAACCTMTATAATGCATTGATCATGCATGTAAGCC & Ahmed et al. (2019), Ann. Bot.123-7, 1231-1251 \\
\hline 9_144478 & AATTCTCAATTCYRTAAAGATGATTTACATGACCAAATCAAATGAGTGGA[A/T]TATTTACWGAGTTCMACKGTCTCTCATTTCTTRAAATTCTAAGCAAAGCA & Ollitrault et al. (2012), BMC Genomics $13: 593$ \\
\hline 9_177279 & ACCTACCGCCGTTTATGCAGCAACTGTGTCTTGAAAACACACCAAGGACT[A/C]TTTTGCCCAATWTGTCTCCAAGTCTATGACCCGTCACCACCTCCGCCTAA & Ahmed et al. (2019), Ann. Bot.123-7, 1231-1251 \\
\hline 9_231920 & ACAGAAGGTGGCTTATAGAAACATGAGATCATCACTGGTATACAGGCCTG[T/C]AATGCTCTCAATGGTGGCCATTGCAGCTGTCTGTGTTTGCGTGGCACTGC & Ahmed et al. (2019), Ann. Bot.123-7, 1231-1251 \\
\hline 9_404236 & TCGGTGTTGGGGCTGAGTTTTGGTGAAGAAATGGAGAGCTTGTCAATCAT[A/G]ACGCCTTCGCATCCATCAAACTTCATGTGAAACTGAGGACTGTTTTGAAT & New \\
\hline 9_1334064 & TAAACAAAACATGCCTCCAAACTTTGGCATAGATCATTAGATCCTTGATA[T/A]GTGTACAGATGGATCAGATGCTTTGTAAATGCAACTCCTGCACCCCAAAA & Ahmed et al. (2020),Front. Plant Sci., fpls.2020.00939 \\
\hline 9_13931363 & GTTTATCTCTTGGGGAAGCAGAGGTGGTGGCTAAGCCCAATTCTTCAAT[T/G]TTTAAGAAAATTGTTGTCAATTATGCCCRCAGCTTTCTTCGGAAAAATTT & Curk et al. (2015), PlosOne 10 (5): e0125628 \\
\hline 9_14749302 & CTTCATCATCACCATCATCAGCGGCAGCAGCAGCAGCAAGCAGGCAACTT[A/C]TCTCAGCAAAATCAACAAAGCATAAAGCTCCAGCTTCGTCTTCTTTTACA & Ahmed et al. (2019), Ann. Bot.123-7, 1231-1251 \\
\hline 9_16069488 & GGTCWTTGTTGGAAACCCACCATATCTTTGTGCATTGACATCTTCCAAGT[A/C]ATAAATATTTTTTGTTTCTGAGCTGTTGTGTGACTCTGCAATAGCTGCCT & Ahmed et al. (2019), Ann. Bot.123-7, 1231-1251 \\
\hline 9_16250199 & AATACAAATCTAATGCAAGTCCAGATCAAGCCACATTGGGTATATTTCTA[C/G]GAAGAGAACATCGTATTGCATAACATGTGAAATATGGAAGGACGATATAA & New \\
\hline 9_18495356 & GAGGATGGTGGCATGTGACATTTGTGAGGTGTGGCAGCACACGCGTTGCC[A/T]TGGCATTGAAGATTCTGGAACTGTGCCACCACTGTTTGTTTGCCCAAGGT & Ahmed et al. (2019), Ann. Bot.123-7, 1231-1251 \\
\hline 9_26923047 & CTGCTCTACGAGTACATGCCGAATGGGAATTTAGACGATTTATTGCACGC[A/C]AAAAATAAAGGAGAAAATTTGGTGGCCGATTGGGTGACGAGGTACAAGAT & New \\
\hline 9_29647599 & GCCTGTTGAATATACAGCAGCAATCCTTTTCTACAGCAATAATGGTCACC[G/A]CGCTGATGGTGCCGAAATGTCTCAACGCCTAAAGGCAGCATTACCAGAAA & Ahmed et al. (2019), Ann. Bot.123-7, 1231-1251 \\
\hline 9_30507813 & AGAGTTGAGTCAGATGGAGATAGAGGAAGATCTCAGTCACGTCAGGCAAA[T/C]AGACGGAGAACTTCTCCCAGCACCAGCTCACATGGCTGCACAGATGAGTA & Ahmed et al. (2019), Ann. Bot.123-7, 1231-1251 \\
\hline 9_30666844 & ACGCGCCAGATCCAGCTGGTCGTACGGATCCGTCGGTATCACCGCCAGTA[T/C]CTCGTCAGGTAAATGAAAGTCGGGCCCACCGCCRCTCTCTCCGCTGCTCT & Ahmed et al. (2019), Ann. Bot.123-7, 1231-1251 \\
\hline 9_31143176 & TCAAATGCATTTTCMACTTTCTTGTGCAAGAAGCTTTTGAAGTTRAAACC[G/A]GAACCAGATTCTTCCTCTTCATCACGCCTTGTGCTCTCCCCAATCTGYTC & Curk et al. (2015), PlosOne 10 (5): e0125628 \\
\hline
\end{tabular}


Table S3. Genotyping results of 27 citrus with the 135 DSNPs.

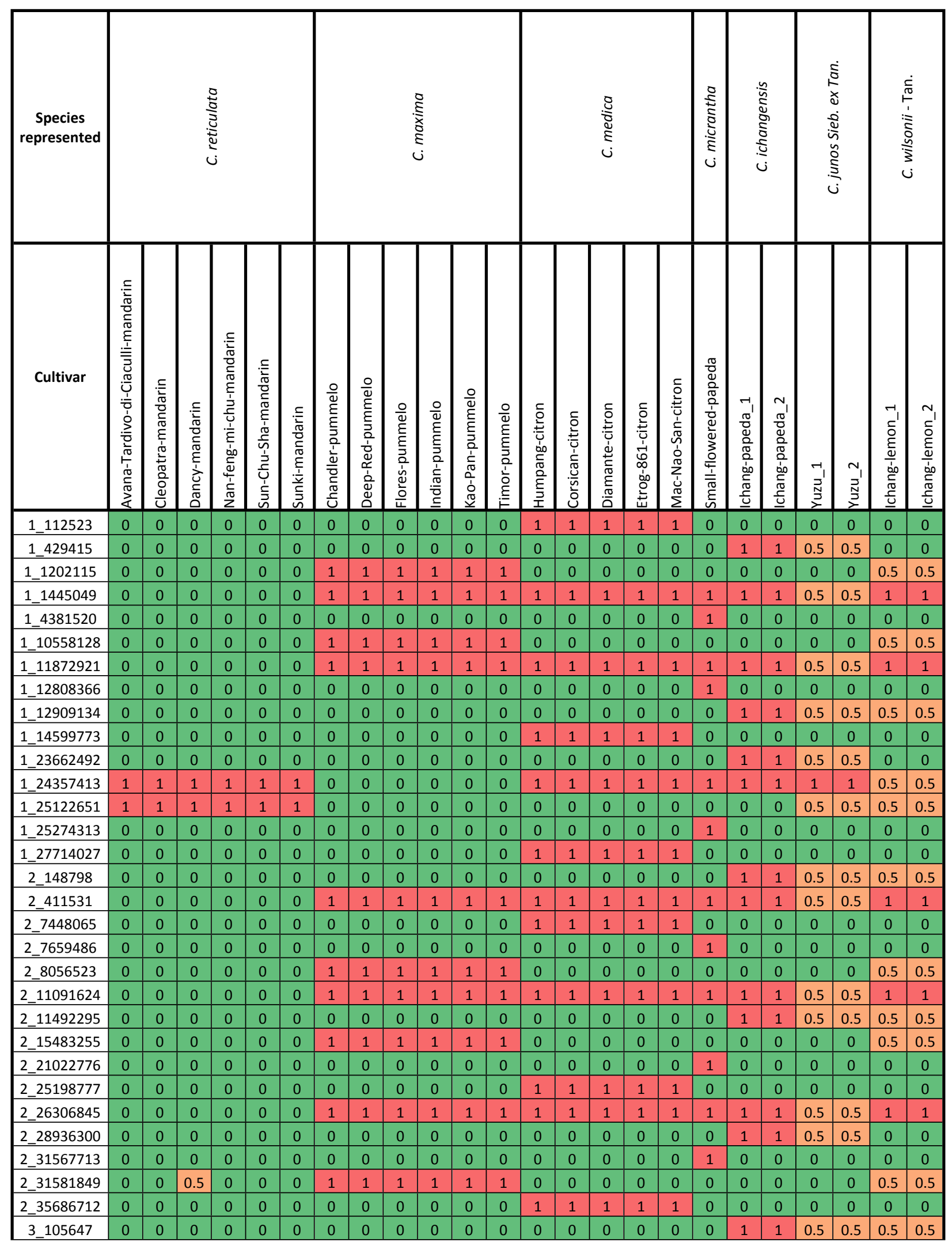




\begin{tabular}{|c|c|c|c|c|c|c|c|c|c|c|c|c|c|c|c|c|c|c|c|c|c|c|c|c|}
\hline 89 & .5 & 0 & 5 & 0 & 0 & 0 & 1 & 1 & 1 & & & & 0 & $\underline{0}$ & 0 & 0 & & & 0 & 0 & 0 & 0 & 0.5 & 0.5 \\
\hline 3_2073865 & 0 & 0 & 0 & 0 & 0 & 0 & 0 & 0 & 0 & & & 0 & 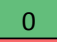 & & & & & & 0 & & 0 & 0 & 0 & 0 \\
\hline 6407 & 0 & 0 & 0 & 0 & 0 & 0 & 1 & 1 & 1 & & & 1 & & & & & & & 1 & 1 & 0.5 & 0.5 & 1 & 4 \\
\hline 3_11355960 & 0 & 0 & 0 & 0 & 0 & 0 & 0 & 0 & 0 & & 0 & 0 & 1 & & 1 & 1 & & & 0 & 0 & 0 & 0 & 0 & 0 \\
\hline 567 & 0 & 0 & 0 & 0 & 0 & 0 & 0 & 0 & 0 & & 0 & 0 & 0 & 0 & 0 & 0 & & & 0 & 0 & 0 & 0 & 0 & 0 \\
\hline 398 & 0 & 0 & 0 & 0 & 0 & 0 & 0 & 0 & 0 & & 0 & 0 & 1 & & 1 & 1 & & & 0 & 0 & 0 & 0 & 0 & 0 \\
\hline 394 & 0 & 0 & 0 & 0 & 0 & 0 & 1 & 1 & 1 & & 1 & 1 & 0 & 0 & 0 & 0 & 0 & & 0 & 0 & 0 & 0 & 0.5 & 0.5 \\
\hline 2884 & 0 & 0 & 0 & 0 & 0 & 0 & 1 & 1 & 1 & & 1 & 1 & 1 & 1 & 1 & 1 & & 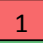 & 1 & 1 & 0.5 & 0.5 & 1 & 1 \\
\hline 3_29381066 & 0 & 0 & 0 & 0 & 0 & 0 & 0 & 0 & 0 & 0 & 0 & 0 & 0 & 0 & 0 & 0 & 0 & c & 1 & 1 & 0.5 & 0.5 & 0.5 & 0.5 \\
\hline 2613 & 0 & 0 & 0 & 0 & 0 & 0 & 0 & 0 & 0 & 0 & 0 & 0 & 0 & 0 & 0 & 0 & 0 & 1 & 0 & 0 & 0 & 0 & 0 & 0 \\
\hline 958 & 0 & 0 & 0 & 0 & 0 & 0 & 1 & 1 & 1 & 1 & 1 & 1 & 1 & 1 & 1 & 1 & 1 & 1 & 1 & 1 & 0.5 & 0.5 & 1 & 1 \\
\hline 150 & 0 & 0 & 0 & 0 & 0 & 0 & 0 & 0 & 0 & 0 & 0 & 0 & 1 & 1 & 1 & 1 & 1 & c & 0 & 0 & 0 & 0 & 0 & 0 \\
\hline 8586 & 0 & 0 & 0 & 0 & 0 & 0 & 1 & 1 & 1 & 1 & 1 & 1 & 0 & U & 0 & 0 & U & C & & 0 & 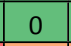 & 0 & 0.5 & 0.5 \\
\hline $3 \_50$ & 0 & 0 & 0 & 0 & 0 & 0 & 0 & 0 & 0 & 0 & 0 & 0 & 0 & & 0 & 0 & & & 1 & 1 & 0.5 & \begin{tabular}{|l|} 
\\
\end{tabular} & 0 & 0 \\
\hline 4_1 & 0 & 0 & 0 & 0 & 0 & 0 & 0 & 0 & 0 & $\underline{0}$ & 0 & 0 & 0 & & 0 & 0 & & & 0 & 0 & 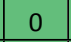 & 0 & 0 & 0 \\
\hline 14 & 0 & 0 & 0 & 0.5 & 0 & 0 & 1 & 1 & 1 & & 1 & 1 & 0 & & 0 &  & & 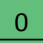 & 0 & 0 & & 0 & \begin{tabular}{|l|}
0.5 \\
\end{tabular} & 0.5 \\
\hline 23 & 0 & 0 & 0 & c & 0 & 0 & 0 & 0 & 0 & 0 & 0 & 0 & 1 & & 1 & $\mathrm{~N}$ & & & & 0 & 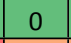 & & 0 & 0 \\
\hline 314 & 0 & 0 & 0 & 0 & 0 & 0 & 0 & 0 & 0 & C & 0 & 0 & 0 & & 0 & 0 & 0 & & 1 & 1 & 0.5 & 0.5 & 0 & 0 \\
\hline 736 & 0 & 0 & 0 & \begin{tabular}{|l|l} 
\\
\end{tabular} & 0 & 0 & 1 & 1 & 1 &  & 1 & 1 & 1 & & 1 & 1 & & & 0 & 0 & 0.5 & \begin{tabular}{|l|}
0.5 \\
\end{tabular} & \begin{tabular}{|l|l}
0.5 \\
\end{tabular} & 0.5 \\
\hline 300 & 0 & 0 & 0 & 0 & 0 & 0 & 1 & 1 & 1 & & 1 & 1 & 1 & 1 & 1 & 1 & 1 & & 1 & 1 & 0.5 & 0.5 & 1 & 1 \\
\hline 632 & 0 & 0 & 0 & 0 & 0 & 0 & 0 & 0 & 0 & c & 0 & 0 & 0 & 0 & 0 & 0 & 0 & & c & 0 & 0 & c & 0 & 0 \\
\hline 117 & 0 & 0 & 0 & 0 & 0 & E & 1 & 1 & 1 & & 1 & 1 & 0 & 0 & 0 & 0 & 0 & ( & C & 0 &  & 0 & \begin{tabular}{|l|}
0.5 \\
\end{tabular} & 0.5 \\
\hline 521 & 0 & 0 & 0 & 0 & 0 & 0 & 0 & 0 & 0 & 0 & 0 & 0 & 0 & 0 & 0 & 0 & 0 & 0 & - & 1 & 0.5 & 0.5 & \begin{tabular}{|l|} 
\\
\end{tabular} & 0.5 \\
\hline 4 & 0 & 0 & 0 & 0 & 0 & 0 & 0 & 0 & 0 & 0 & 0 & 0 & 1 & \pm & 1 & 1 & 1 & 0 & 0 & 0 & 0 & 0 & 0 & 0 \\
\hline $4 \_2$ & 0 & 0 & 0 & 0 & 0 & \begin{tabular}{|l|}
0 \\
\end{tabular} & 0 & 0 & 0 & 0 & 0 & 0 & 0 & 0 & 0 & 0 & 0 & 1 & 0 & 0 & C & 0 & 0 & 0 \\
\hline $4 \_2$ & 0 & 0 & 0 & 0 & 0 & - & 1 & 1 & 1 & 1 & 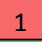 & 1 & 0 & 0 & 0 & 0 & 0 & $c$ & 1 & 0 & 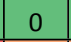 & 0 & \begin{tabular}{|l|}
0.5 \\
\end{tabular} & 0.5 \\
\hline 4 & 0 & 0 & 0 & 0 & 0 & 0 & 0 & 0 & 0 & 0 & 0 & 0 & 0 & 0 & 0 & 0 & 0 & c & & 1 & 0.5 & 0.5 & 0.5 & 0.5 \\
\hline 853 & 0 & 0 & 0 & 0 & 0 & 0 & 0 & 0 & 0 & 0 & 0 & c & 1 & & 1 & 1 & 1 & & & 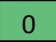 & & 0 & 0 & 0 \\
\hline 9957 & 0 & 0 & 0 & 0 & 0 & 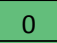 & 1 & 1 & 1 & 1 & 1 & 1 & 1 & 1 & 1 & 1 & 1 & 1 & 1 & 1 & 0.5 & \begin{tabular}{|l|}
0.5 \\
\end{tabular} & 0.5 & \begin{tabular}{|l} 
\\
\end{tabular} \\
\hline $5 \_1$ & 0 & 0 & 0 & 0 & 0 & 0 & \begin{tabular}{|l|}
0.5 \\
\end{tabular} & 1 & 1 & 1 & 1 & 0 & 0 & 0 & 0 & 0 & 0 & C & 0 & 0 & 0.5 & \begin{tabular}{|l|}
0.5 \\
\end{tabular} & \begin{tabular}{|l}
0.5 \\
\end{tabular} & 0.5 \\
\hline 5_4 & 0 & 0 & 0 & 0 & 0 & 0 & 0 & 0 & 0 & 0 & 0 & 0 & 1 & 1 & 1 & 1 & 1 & ( & 0 & 0 & 0 & 0 & 0 & 0 \\
\hline 5_6729832 & 0 & 0 & 0 & 0 & 0 & 0 & 1 & 1 & 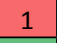 & 1 & 1 & 1 & 1 & 1 & 1 & 1 & 1 & & 0 & 0 & & 0 & 0.5 & 0.5 \\
\hline 3417 & 0 & 0 & 0 & 0 & 0 &  & 0 & 0 & 0 & $\underline{0}$ & 0 & 0 & 0 & 0 & 0 & 0 & 0 & c & 1 & 4 & & 0 & 0 & 0 \\
\hline 6308 & 0 & 0 & 0 & 0 & 0 & 0 & 0 & 0 & 0 & & 0 & 0 & 0 & 0 & 0 & 0 & 0 & $=$ & 0 & 0 & 0 & 0 & 0 & 0 \\
\hline 2485 & 0 & 0 & 0 & 0 & 0 & 0 & 1 & 1 & 1 & & 1 & 1 & 1 & 1 & 1 & 1 & 1 & 1 & 1 & 1 & 0.5 & 0.5 & 1 & 1 \\
\hline 647 & 0 & 0 & 0 & 0 & 0 & 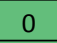 & 0 & 0 & 0 & 0 & 0 & 0 & 1 & 1 & 1 & 1 & 1 & c & 0 & 0 & 0 & 0 & 0 & 0 \\
\hline 312 & 0 & 0 & 0 & 0 & 0 & 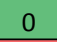 & 0 & 0 & 0 & & 0 & 0 & 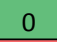 & 0 & 0 & 0 & 0 & 0 & 1 & 1 & 0.5 & 0.5 & 0.5 & 0.5 \\
\hline 907 & 1 & 1 & 1 & 1 & 1 & 1 & 0 & 0 & 0 & 0 & 0 & 0 & 1 & 1 & 1 & 1 & + & 1 & 1 & 1 & 1 & 1 & 0.5 & 0.5 \\
\hline 626 & 0 & 0 & 0 & 0 & 0 & 0 & 0 & 0 & 0 & 0 & 0 & 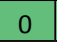 & 0 & 0 & 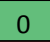 & 0 & 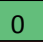 & 1 & 0 & 0 & 0 & 0 & 0 & 0 \\
\hline 150 & 0 & 0 & 0 & 0 & 0 & 0 & 1 & 1 & 1 & 1 & 4 & 1 & 1 &  & 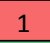 & 1 & 1 & 1 & 1 & 1 & 0.5 & 0.5 & 1 & 1 \\
\hline 357 & 0 & 0 & 0 & 0 & 0 & 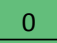 & 1 & 1 & 1 & 1 & 1 & 1 & 0 & 0 & 0 & 0 & 0 & 0 & 0 & 0 & 0 & 0 & \begin{tabular}{|l|}
0.5 \\
\end{tabular} & 0.5 \\
\hline & 0 & 0 & 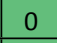 & 0 & 0 & 0 & 0 & 0 & 0 & 0 & 0 & 0 & 0 & 0 & 0 & 0 & 0 & c & 1 & 1 & 0.5 & 0.5 & 0.5 & 0.5 \\
\hline & 0 & 0 & 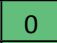 & 0 & 0 & 0 & 0 & 0 & 0 & $\underline{0}$ & 0 & 0 & 1 & 1 & 1 & 1 & 1 & 0 & 0 & 0 & & & 0 & 0 \\
\hline 502 & 0 & 0 & 0 & 0 & 0 & 0 & 0 & 0 & 0 & $\underline{0}$ & 0 & 0 & 0 & 0 & 0 & 0 & 0 & 1 & 0 & 0 & 0 & 0 & 0 & 0 \\
\hline & 0 & 0 & 0 & 0 & 0 & 0 & 0 & 0 & 0 & $\underline{0}$ & 0 & 0 & 0 & 0 & 0 & 0 & 0 & 0 & 1 & 1 & 0.5 & 0.5 & 0 & 0 \\
\hline 988 & 0 & 0 & 0 & 0 & 0 & 0 & 1 & 1 & 1 & 1 & 1 & 1 & 0 & 0 & 0 & 0 & 0 & 0 & 1 & 1 & 0.5 & 0.5 & 0.5 & 0.5 \\
\hline & 0 & 0 & 0 & 0 & 0 & 0 & 0 & 0 & 0 & 0 & 0 & 0 & 1 & 1 & 1 & 1 & 1 & 0 & 0 & 0 & & 0 & 0 & 0 \\
\hline 346 & 0 & 0 & 0 & $\underline{0}$ & 0 & 0 & 0 & 0 & 0 & $\underline{0}$ & 0 & 0 & 0 & 0 & 0 & 0 & 0 & 1 & 0 & 0 & 0 & 0 & 0 & 0 \\
\hline 009 & 0 & 0 & 0 & 0 & 0 & 0 & 1 & 1 & 1 & 1 & 1 & 1 & 1 & 1 & 1 & 1 & 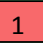 & 1 & 1 & 1 & \begin{tabular}{|l|}
0.5 \\
\end{tabular} & \begin{tabular}{|l|}
0.5 \\
\end{tabular} & 0.5 & 0.5 \\
\hline 5273 & 0 & 0 & 0 & 0 & 0 & 0 & 1 & 1 & 1 & 1 & 1 & 1 & 0 & 0 & 0 & 0 & 0 & 0 & 0 & 0 & 0 & 0 & 0.5 & 0.5 \\
\hline 009 & 0 & 0 & 0 & 0 & 0 & 0 & 0 & 0 & 0 & 0 & 0 & 0 & 0 & 0 & 0 & 0 & 0 & 0 & 1 & 1 & \begin{tabular}{|l|}
0.5 \\
\end{tabular} & \begin{tabular}{|l|}
0.5 \\
\end{tabular} & 0 & 0 \\
\hline 033 & 0 & 0 & 0 & 0 & 0 & 0 & 1 & 1 & 1 & 1 & 1 & 1 & 1 & 1 & 1 & 1 & 1 & 1 & 1 & 1 & 0.5 & 0.5 & \begin{tabular}{|l}
0.5 \\
\end{tabular} & 0.5 \\
\hline 1556 & 0 & 0 & 0 & 0 & 0 & 0 & 0 & 0 & 0 & 0 & 0 & 0 & 0 & 0 & 0 & 0 & 0 & 1 & 0 & 0 & 0 & 0 & 0 & 0 \\
\hline 6315 & 0 & 0 & 0 & 0 & 0 & 0 & 0 & 0 & 0 & 0 & 0 & 0 & 1 & 1 & 1 & 1 & 1 & 0 & 0 & 0 & 0 & 0 & 0 & 0 \\
\hline 22952731 & 0 & 0 & 0 & 0 & 0 & 0 & 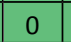 & 0 & 0 & & U & 0 & u & 0 & U & 0 & 0 & 1 & 0 & 0 & 0 & 0 & 0 & 0 \\
\hline
\end{tabular}




\begin{tabular}{|c|c|c|c|c|c|c|c|c|c|c|c|c|c|c|c|c|c|c|c|c|c|c|c|c|}
\hline 6_23700512 & 0.5 & 1 & 1 & 1 & 1 & 1 & 0 & 0 & 0 & 0 & 0 & 0 & 1 & 1 & 1 & 1 & 1 & 1 & 1 & 1 & 1 & 1 & 1 & 1 \\
\hline 6_23938216 & 0 & 0 & 0 & 0 & 0 & 0 & 0 & 0 & 0 & . & 0 & 0 & 0 & 0 & 0 & 0 &  & 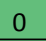 & 1 & & 0.5 & 0.5 & \begin{tabular}{|l|}
0.5 \\
\end{tabular} & 0.5 \\
\hline 6_25003350 & 0.5 & 1 & 1 & 1 & 1 & 1 & 0 & 0 & 0 & 0 & 0 & 0 & 0 & 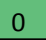 & 0 & 0 & 0 & 0 & 0 & 0 & 0.5 & 0.5 & 0 & 0 \\
\hline 6_25003653 & 0 & 0 & 0 & 0 & 0 & 0 & 0 & 0 & 0 & 0 & 0 & 0 & 1 & 1 & 1 & 1 & 1 & 0 & 0 & 0 & 0 & 0 & 0 & 0 \\
\hline 7_137886 & 0 & 0 & 0 & 0 & 0 & 0 & 0 & 0 & 0 & 0 & 0 & 0 & 1 & 1 & 1 & 1 & 1 & 0 & 0 & 0 & 0 & 0 & 0 & 0 \\
\hline 7_355705 & 0 & 0 & 0 & 0 & 0 & 0 & 0 & 0 & 0 & 0 & 0 & 0 & 0 & 0 & 0 & 0 &  & 0 & 1 & 1 & 0.5 & 0.5 & 0 & 0 \\
\hline 7_2639448 & 0 & 0 & 0 & 0 & 0 & 0 & 1 & 1 & 1 & 1 & 1 & 1 & 1 & 1 & 1 & 1 & 1 & 1 & 1 & 1 & 0.5 & 0.5 & 0.5 & 0.5 \\
\hline 7_2689161 & 0 & 0 & 0 & 0 & 0 & 0 & 1 & 1 & 1 & 1 & 1 & 1 & 0 & 0 & 0 & 0 & 0 & 0 & 0 & . & 0 & 0 & 0.5 & 0.5 \\
\hline 7_4057807 & 0 & 0 & 0 & 0 & 0 & 0 & 0 & 0 & 0 & 0 & 0 & 0 & 0 & 0 & 0 & 0 & 0 & 1 & 0 & 0 & 0 & 0 & 0 & 0 \\
\hline 7_6084876 & 0 & 0 & 0 & 0 & 0 & 0 & 0 & 0 & 0 & 0 & 0 & 0 & 0 & 0 & 0 & 0 & 0 & 1 & 0 & 0 & 0 & 0 & 0 & 0 \\
\hline 7_8380800 & 0 & 0 & 0 & 0 & 0 & 0 & 1 & 1 & 1 & 1 & 1 & 1 & 1 & 1 & 1 & 1 & 1 & 1 & 1 & 1 & 0.5 & 0.5 & 1 & 1 \\
\hline 7_8928501 & 0 & 0 & 0 & 0 & 0 & 0 & 0 & 0 & 0 & 0 & 0 & 0.5 & 1 & 1 & 1 & 0.5 & 1 & 0 & 0 &  & 0 & 0 & 0 & 0 \\
\hline 7_10698885 & 0 & 0 & 0 & 0 & 0 & 0 & 1 & 1 & 1 & 1 & 1 & 1 & 0 & 0 & 0 & 0 & 0 & 0 & 1 & 1 & 0.5 & 0.5 & 1 & 1 \\
\hline 7_13741299 & 0 & 0 & 0 & 0 & 0 & 0 & 0 & 0 & 0 & 0 & 0 & 0 & 0 & 0 & 0 & 0 & 0 & 0 & 1 & 1 & 0.5 & 0.5 & 0.5 & 0.5 \\
\hline 7_18363367 & 0 & 0 & 0 & 0 & 0 & 0 & 1 & 1 & 1 & 1 & 1 & 1 & 1 & 1 & 1 & 1 & 1 & 1 & 1 & 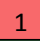 & 0.5 & 0.5 & \begin{tabular}{|l|}
0.5 \\
\end{tabular} & 0.5 \\
\hline 7_19114393 & 0 & 0 & 0 & 0 & 0 & 0 & 0 & 0 & 0 & 0 & 0 & 0 & 1 & 1 & 1 & 1 & 1 & 0 & 0 & 0 & 0 & 0 & 0 & 0 \\
\hline 7_20103116 & 0 & 0 & 0 & 0 & 0 & 0 & 0 & 0 & 0 & 0 & 0 & 0 & 0 & 0 & 0 & 0 & 0 & 1 & 0 & 0 & 0 & 0 & 0 & 0 \\
\hline 7_20899506 & 0 & 0 & 0 & 0 & 0 & 0 & 1 & 1 & 1 & 1 & 1 & 1 & 0 & 0 & 0 & 0 & 0 & 0 & 0 & 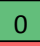 & 0 & 0 & 0.5 & 0.5 \\
\hline 7_21043581 & 0 & 0 & 0 & 0 & 0 & 0 & 0 & 0 & 0 & 0 & 0 & 0 & 0 & 0 & 0 & 0 & 0 & 0 & 1 & 1 & 0.5 & 0.5 & 0 & 0 \\
\hline 8_44391 & 0 & 0 & 0 & 0 & 0 & 0 & 0 & 0 & 0 & 0 & 0 & 0 & 1 & 1 & 1 & 1 & 1 & 0 & 0 & 0 & 0 & 0 & 0 & 0 \\
\hline 8_756476 & 0 & 0 & 0 & 0 & 0 & 0 & 0 & 0 & 0 & 0 & 0 & 0 & 0 & 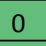 & 0 & 0 & 0 & 0 & 1 & 1 & 0.5 & 0.5 & 0.5 & 0.5 \\
\hline 8_2438682 & 0.5 & 0 & 0 & 0.5 & 0 & 0 & 1 & 1 & 1 & 1 & 1 & 1 & 0 & 0 & 0 & 0 & 0 & 0 & 0 & 0 & 0 & 0 & 0.5 & 0.5 \\
\hline 8_3998031 & 0 & 0 & 0 & 0 & 0 & 0 & 0 & 0 & 0 & 0 & 0 & 0 & 0 & 0 & 0 & 0 & 0 & 1 & 0 & 0 & 0 & 0 & 0 & 0 \\
\hline 8_8442079 & 0 & 0 & 0 & 0 & 0 & 0 & 1 & 1 & 1 & 1 & 1 & 1 & 0 & 0 & 0 & 0 & 0 & 0 & 0 & 0 & 0 & 0 & 0.5 & 0.5 \\
\hline 8_8518384 & 0 & 0 & 0 & 0 & 0 & 0 & 1 & 1 & 1 & 1 & 1 & 1 & 1 & 1 & 1 & 1 & 1 & 1 & 1 & 1 & 0.5 & 0.5 & 1 & 1 \\
\hline 8_12939045 & 0 & 0 & 0 & 0 & 0 & 0 & 0 & 0 & 0 & 0 & 0 & 0 & 0 & 0 & 0 & 0 & 0 & 1 & 0 & 0 & 0 & 0 & 0 & 0 \\
\hline 6495 & 0 & 0 & 0 & 0 & 0 & 0 & 0 & 0 & 0 & 0 & 0 & 0 & 0 & 0 & 0 & 0 & 0 & 0 & 1 &  & 0.5 & 0.5 & 0.5 & 0.5 \\
\hline 8_14340205 & 0 & 0 & 0 & 0 & 0 & 0 & 0 & 0 & 0 & 0 & 0 & 0 & 1 & 1 & 1 & 1 & 1 & 0 & 0 & 0 & 0 & 0 & 0 & 0 \\
\hline 8_17526645 & 0 & 0 & 0 & 0 & 0 & 0 & 1 & 1 & 1 & 1 & 1 & 1 & 1 & 1 & 1 & 1 & 1 & 1 & 1 & 1 & 0.5 & 0.5 & 0.5 & 0.5 \\
\hline 8_23599778 & 0 & 0 & 0 & 0 & 0 & 0 & 1 & 1 & 1 & 1 & 1 & 1 & 0 & 0 & 0 & 0 & 0 & 0 & 0 & 0 & 0 & 0 & 0.5 & 0.5 \\
\hline 8_23989221 & 0 & 0 & 0 & 0 & 0 & 0 & 0 & 0 & 0 & 0 & 0 & 0 & 0 & 0 & 0 & 0 & 0 & 0 & 1 & 1 & 0.5 & 0.5 & 0 & 0 \\
\hline 8_24122489 & 0 & 0 & 0 & 0 & 0 & 0 & 1 & 1 & 1 & 1 & 1 & 1 & 1 & 1 & 1 & 1 & 1 & 1 & 1 & 1 & 0.5 & 0.5 & \begin{tabular}{|l|}
0.5 \\
\end{tabular} & 0.5 \\
\hline 8_24200344 & 0 & 0 & 0 & 0 & 0 & 0 & 0 & 0 & 0 & 0 & 0 & 0 & 0 & 0 & 0 & 0 & 0 & 1 & 0 & 0 & 0 & 0 & 0 & 0 \\
\hline 8_24991815 & 0 & 0 & 0 & 0 & 0 & 0 & 0 & 0 & 0 & 0 & 0 & 0 & 1 & 1 & 1 & 1 & 1 & 0 & 0 & 0 & 0 & 0 & 0 & 0 \\
\hline 9_144478 & 0 & 0 & 0 & 0.5 & 0 & 0 & 1 & 1 & 1 & 1 & 1 & 1 & 1 & 1 & 1 & 1 & 1 & 1 & 1 & 1 & 0.5 & 0.5 & 0.5 & 0.5 \\
\hline 9_177279 & 0 & 0 & 0 & 0 & 0 & 0 & 0 & 0 & 0 & 0 & 0 & 0 & 1 & 1 & 1 & 1 & 1 & 0 & 0 & 0 & 0 & 0 & 0 & 0 \\
\hline 9_231920 & 0 & 0 & 0 & 0.5 & 0 & 0 & 1 & 1 & 1 & 1 & 1 & 1 & 0 & 0 & 0 & 0 & 0 & 0 & 0 &  & 0 & 0 & 0.5 & 0.5 \\
\hline 9_404236 & 0 & 0 & 0 & 0 & 0 & 0 & 0 & 0 & 0 & 0 & 0 & 0 & 0 & 0 & 0 & 0 & 0 & 0 & 1 & 1 & 0.5 & 0.5 & 0 & 0 \\
\hline 9_1334064 & 0 & 0 & 0 & 0 & 0 & 0 & 0 & 0 & 0 & 0 & 0 & 0 & 0 & 0 & 0 & 0 & 0 & 1 & 0 & - & 0 & 0 & 0 & 0 \\
\hline 9_13931363 & 0 & 0 & 0 & 0 & 0 & 0 & 0 & 0 & 0 & 0 & 0 & 0 & 0 & 0 & 0 & 0 & 0 & 1 & 0 &  & 0 & 0 & 0 & 0 \\
\hline 9_14749302 & 0 & 0 & 0 & 0 & 0 & 0 & 0 & 0 & 0 & 0 & 0 & 0 & 1 & 1 & 1 & 1 & 1 & 0 & 0 & 0 & 0 & 0 & 0 & 0 \\
\hline 9_16069488 & 0 & 0 & 0 & 0 & 0 & 0 & 1 & 1 & 1 & 1 & 1 & 1 & 0 & 0 & 0 & 0 & 0 & 0 & 0 & 0 & 0 & 0 & \begin{tabular}{|l|}
0.5 \\
\end{tabular} & 0.5 \\
\hline 9_16250199 & 0 & 0 & 0 & 0 & 0 & 0 & 0 & 0 & 0 & 0 & 0 & 0 & 0 & 0 & 0 & 0 & 0 & 0 & 1 & 1 & 0.5 & 0.5 & 0.5 & 0.5 \\
\hline 9_18495356 & 0 & 0 & 0 & 0 & 0 & 0 & 1 & 1 & 1 & 1 & 1 & 1 & 1 & 1 & 1 & 1 & 1 & 1 & 1 & 1 & 0.5 & 0.5 & 1 & 1 \\
\hline 9_26923047 & 0 & 0 & 0 & 0 & 0 & 0 & 0 & 0 & 0 & 0 & 0 & 0 & 0 & 0 & 0 & 0 & 0 & 0 & 1 & 1 & 0.5 & 0.5 & 0.5 & 0.5 \\
\hline 9_29647599 & 0 & 0 & 0 & 0 & 0 & 0 & 1 & 1 & 1 & 1 & 1 & 1 & 0 & 0 & 0 & 0 & 0 & 0 & 0 & 0 & 0 & 0 & 0.5 & 0.5 \\
\hline 9_30507813 & 0 & 0 & 0 & 0 & 0 & 0 & 0 & 0 & 0 & 0 & 0 & 0 & 1 & 1 & 1 & 1 & 1 & 0 & 0 & 0 & 0 & 0 & 0 & 0 \\
\hline 9_30666844 & 0 & 0 & 0 & 0 & 0 & 0 & 1 & 1 & 1 & 1 & 1 & 1 & 1 & 1 & 1 & 1 & 1 & 1 & 1 & 1 & 0.5 & 0.5 & 0.5 & 0.5 \\
\hline 9_31143176 & 0 & 0 & 0 & 0 & 0 & 0 & 0 & 0 & 0 & 0 & 0 & 0 & 0 & 0 & 0 & 0 & 0 & 1 & 0 & 0 & 0 & 0 & 0 & 0 \\
\hline
\end{tabular}

Genotype coding:

0 : homozygous for the reference allele of $\mathrm{C}$. clementina reference genome

1: homozygous for the alternative allele

0.5 : heterozygous

$\mathrm{N}$ : Not determined 
Figure S1. MS spectra of 3-methyl-3-sulfanylbutyl acetate obtained by GC-QTOF-MS.




Figure S2. Infrared spectrum of 3-methyl-3-sulfanylbutyl acetate obtained by GC-FTIR.

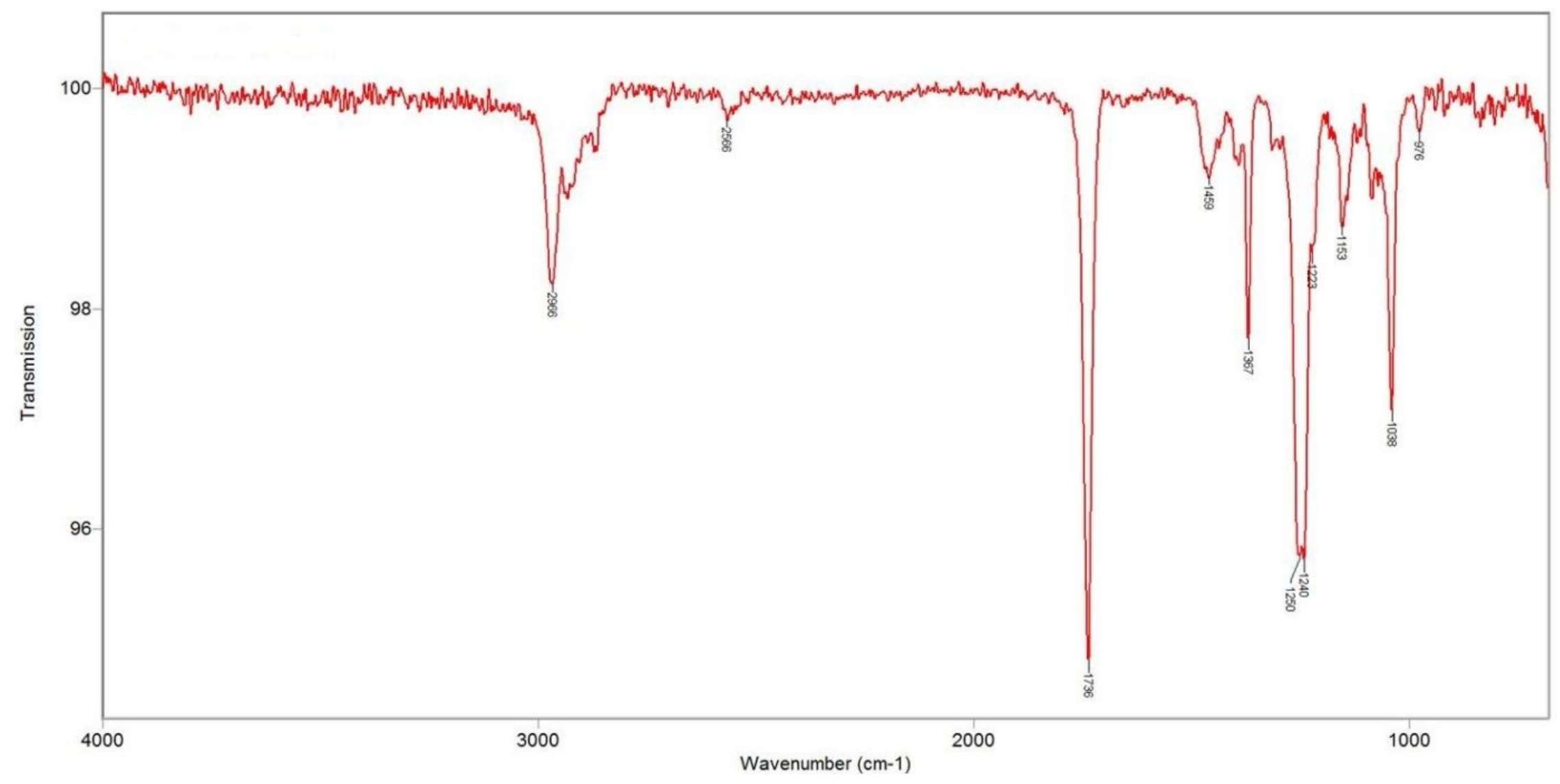

